\title{
Highlights in Mesoporous Silica Nanoparticles as a Multifunctional Controlled Drug Delivery Nanoplatform for Infectious Diseases Treatment
}

\author{
Gabriela Corrêa Carvalho' - Rafael Miguel Sábio' (1) - Tais de Cássia Ribeiro' . \\ Andreia Sofia Monteiro $^{2}$ - Daniela Vassalo Pereira ${ }^{2}$. Sidney José Lima Ribeiro ${ }^{2} \cdot$ Marlus Chorilli ${ }^{1}$
}

Received: 30 June 2020 / Accepted: 25 August 2020 / Published online: 7 September 2020

(C) Springer Science+Business Media, LLC, part of Springer Nature 2020

\begin{abstract}
Infectious diseases are a major global concern being responsible for high morbidity and mortality mainly due to the development and enhancement of multidrug-resistant microorganisms exposing the fragility of medicines and vaccines commonly used to these treatments. Taking into account the scarcity of effective formulation to treat infectious diseases, nanotechnology offers a vast possibility of ground-breaking platforms to design new treatment through smart nanostructures for drug delivery purposes. Among the available nanosystems, mesoporous silica nanoparticles (MSNs) stand out due their multifunctionality, biocompatibility and tunable properties make them emerging and actual nanocarriers for specific and controlled drug release. Considering the high demand for diseases prevention and treatment, this review exploits the MSNs fabrication and their behavior in biological media besides highlighting the most of strategies to explore the wide MSNs functionality as engineered, smart and effective controlled drug release nanovehicles for infectious diseases treatment.
\end{abstract}

KEY WORDS biological behavior · drug delivery · infectious diseases · mesoporous silica nanoparticles · multifunctional nanocarriers

Gabriela Corrêa Carvalho and Rafael Miguel Sábio contributed equally to this work.

Rafael Miguel Sábio

rafaelmsabio@gmail.com

School of Pharmaceutical Sciences, São Paulo State University (UNESP), Araraquara 14800-903, Brazil

2 Institute of Chemistry, São Paulo State University (UNESP), Araraquara 14800-060, Brazil

\section{INTRODUCTION}

Infectious diseases are increasingly emerging as a cause of morbidity and mortality worldwide, mainly due to the fact that some diseases are caused by multi-resistant microorganisms to existing drugs $(1,2)$. Antimicrobial resistance occurs when microorganisms (such as bacteria, fungi, viruses and parasites) change when they are exposed to antimicrobial drugs (such as antibiotics, antifungals, antivirals, antimalarials, and anthelmintics). As a result, the medicines become ineffective and infections persist in the body, increasing the risk of spread to others. Without effective antimicrobials for prevention and treatment of infections, medical procedures such as organ transplantation, cancer chemotherapy, diabetes management and major surgery (for example, caesarean sections or hip replacements) become very high risk. Beyond that, antimicrobial resistance increases the cost of health care with lengthier stays in hospitals and more intensive care required, as stated by World Health Organization (WHO) $(1,3)$.

Considering the health concern, nanotechnology offers potential opportunities to design effective medicines and vaccines for diseases treatment (4-6). Nanotechnology is a multidisciplinary science field that studies the fabrication and application of structures with one of its dimensions less than $100 \mathrm{~nm}(7,8)$. The use of nanostructures in health area are desirable due to the fact that their size are 100 to 10000 times smaller than eukaryotic cells and similar size to the most biological molecules and structures including DNA, proteins, cell membranes and pathogenic microorganisms $(9,10)$. In addition, the nanometric size can potentialize nanomaterials action and efficacy comprising an emerging tools to develop therapeutic agents, diagnosis systems, medical devices and drug delivery platforms including medicines and vaccines for infectious diseases treatment (8,11-14).

The use of nanomaterials as carriers for controlled drug release has increasingly been explored due to their small size, high surface area and ability to pass through epithelial and 
endothelial barriers increasing drugs permeability without change its pharmacological properties (15-18). These nanosystems can reach the action site with considerable drugs concentration increasing their specific retention time reducing side effects (8,15-19). Nanoparticles can be produced from various materials such as amphiphilic structures, lipids, biodegradable polymers and inorganic compounds $(1,2,16,20)$. Specifically, inorganic platforms as mesoporous silica nanoparticles (MSNs) comprise a potential nanosystem for healthcare applications due to interesting characteristics such as easy functionalization, large surface area, high chemical and physical stabilities and hydroxyl groups available in their surface/ pores. In addition, MSNs tunable morphology, size, surface charges and pores make them carriers with good biocompatibility and biodegradability, low toxicity, good biodistribution and elimination (12,21-27). Drugs loaded into MSNs pores leading to pharmacological properties maintenance besides protection against degradation and immediate drug release $(23,24,28,29)$.

Taking into account that infectious diseases are a serious global public health concern and MSNs present several interesting properties that make them widely desirable to design controlled drug delivery systems, this review reports a brief overview of MSNs properties and their influence in biological behavior as well as highlights the advances in MSNs applications as multifunctional controlled drug release nanoplatforms for infectious diseases treatment.

\section{INFECTIOUS DISEASES}

Infectious diseases are those caused by pathogenic microorganisms such as bacteria, fungi, viruses and parasites, which invade host's cells in order to reproduce and thereby damage tissues causing disease. These diseases are transmitted directly or indirectly from person to person (30-32). Currently, infectious diseases are emerging at a global level and a rate never seen before, representing a challenge for the 21 st century regarding to the discovery and development of new formulations $(31,33)$.

The WHO brings cholera, Ebola virus, influenza, meningitis, COVID-19, poliomyelitis, yellow fever as public health emergencies. Five of them are caused by virus and two by bacteria (considering that the main meningitis causing agent is bacteria) (34). This may explain the data obtained in Fig. 1, where the association of the keywords "infectious diseases and virus and new formulations" recovered the largest number of manuscripts published in the period from 2000 to 2020, corresponding to $41 \%$, followed by the association of the keywords "infectious diseases and bacteria and new formulations" which are assigned to $28 \%$.

Because virus cannot reproduce or shows metabolism outside a host cell, it is not considered an organism, but rather particles, which are coated with a protein or lipid capsid. As for the genetic material, they are divided into double or single stranded DNA viruses, or RNA, single or double stranded viruses (30). Viruses listed as a public health emergency by the WHO, Ebola virus, influenza, COVID-19, poliomyelitis and yellow fever, are all single stranded RNA viruses (35-41).

Coronavirus, which causes COVID-19, is the current major threat due to its ability to spread quickly $(35,42)$. Until May 31, 2020 5,956,883 confirmed cases (being that the largest number of cases are concentrated in the Americas) and 366,409 deaths worldwide, which shows the seriousness of this pandemic started in December 2019. Generally, this virus causes infection in the upper respiratory tract, or, in some patients in the lower respiratory tract, presenting fever, cough and headache as main symptoms $(6,34,35,42,43)$.

In the period of 2014-2016, worldwide efforts were focused on combating the outbreak caused by Ebola virus in West Africa, a severe hemorrhagic fever, fatal in many cases, the average mortality rate in this period was $50 \%$. In addition to the great local concern, the world community feared for dissemination throughout the world and for its use as a biological weapon. Although the outbreak period has passed, there is currently a great concern related to the persistence of this virus in the reproductive tract of male people who survived this disease $(34,37,44)$.

The yellow fever virus, transmitted by mosquitoes of the genera Haemagogus and Aedes, was the cause of severe epidemics that occurred in the $17 \mathrm{th}, 18$ th and 19th centuries. In the 1930 s, fundamental vaccines were developed to contain this disease, but it is not the same that is used nowadays due to the appearance of adverse reactions. Even with the vaccine, this disease is still epidemic in many regions such as Africa and South America. It is a severe acute disease that presents as symptoms fever, epigastric pain, nausea, vomiting, renal failure, hepatitis accompanied by jaundice, hemorrhage, shock and death in $20-60 \%$ of cases $(34,40,45,46)$.

In the years 1918 and 1919, the influenza virus was the cause of a pandemic that caused about 20 to 50 million fatal victims worldwide (47). This pandemic causing strain was the influenza virus type A, which has the subtypes H1N1, H3N2 and $\mathrm{H} 5 \mathrm{N1}$, although influenza virus type $\mathrm{B}$ and type $\mathrm{C}$ are also infectious in humans $(41,48)$. Nowadays, the major concern consists on prevents and controls this seasonal disease in order to avoid new influenza pandemics from happening (34).

Poliomyelitis is a disease that has been plaguing humanity for centuries, its first clinical description dates from 1789, being that only in 1955 the first vaccine was developed (49). Children are the most affected by this disease, which can affect the nervous system and cause paralysis. Nowadays, it is endemic in areas of Afghanistan and Pakistan, and in the period of 10 years, 200,000 new cases were observed, probably due to failures in strategic actions implementation, leading to a high transmission of this virus (34). 
Fig. I Percentage of new formulations research in the infectious diseases field. The numbers of manuscripts were retrieved from SCOPUS $\AA$ database by searching "infectious diseases/virus/new formulations", "infectious diseases/bacteria/new formulations", "infectious diseases/ parasites/new formulations" and "infectious diseases/fungi/new formulations" (Data accessed June 0I, 2020).

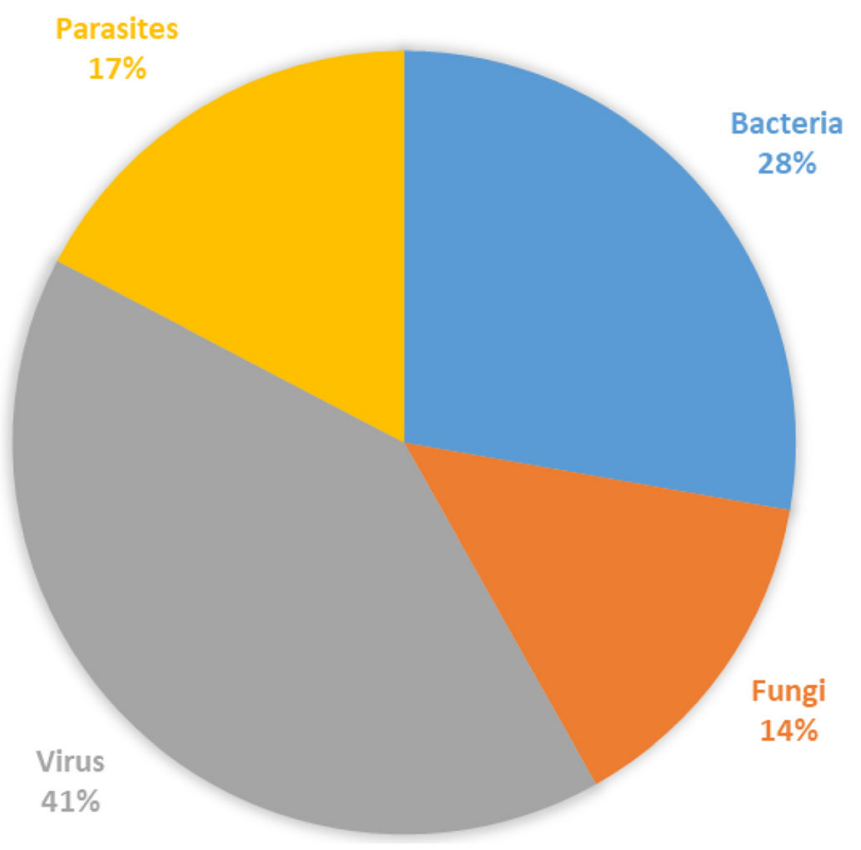

As for parasitic diseases, they are caused by protozoa (single-celled eukaryotic cells) and helminths (invertebrate animals) (30,55). Parasitic diseases are a public health concern that affects humans throughout history, leading to the millions death of people annually (56,57).

Faced with the human being fragility in relation to the severity of some infectious diseases that prove to be a worldwide public health problem, the current scenario in which the planet finds itself sparing no effort in order to face a pandemic or even in the fight against resistant microorganisms, considering the new formulations development essential. In light of this, the drugs association with promising nanocarriers such as MSNs is fundamental to achieve new smart nanotherapeutics systems for all aforementioned diseases (12,20,58-60).

\section{MESOPOROUS SILICA NANOPARTICLES}

\section{Synthesis and Types of Mesoporous Silica Nanoparticles}

Need for materials with high thermal, chemical and mechanical properties have led to the development of MSNs. As per IUPAC, mesoporous materials are define as the one having a pore size in the range of $2-50 \mathrm{~nm}$ and an ordered arrangement of pores giving an ordered structure to it $(61,62)$. Mobil Research and Development Corporation was the first to synthesize mesoporous solids from aluminosilicate gels using liquid crystal template mechanism in the year 1992. They designated it as (Mobil Crystalline Materials or Mobil Composition of Matter) MCM-41. The pore size of the mesoporous materials could be varied and tuned through the choice of surfactants used. MCM-41 is hexagonal with a pore 
diameter from 2.5 to $6 \mathrm{~nm}$ wherein cationic surfactants were used as templates. Various other materials of mesoporous nature have also been synthesized by varying the starting precursors and reaction conditions. These mesoporous materials may vary in their structural arrangement or the pore size. MCM-48 has a cubic arrangement whereas MCM-50 has a lamella-like arrangement $(61,63)$. Non-ionic triblock copolymers like alkyl poly(ethylene oxide) (PEO) oligomeric surfactants and poly(alkylene oxide) block copolymers have also been used by University of California, Santa Barbara for synthesized the named Santa Barbara Amorphous materials (SBA). The ratio of ethylene oxide to propylene oxide was varied to achieve the desired symmetry of mesoporous materials (SBA-11 (cubic), SBA-12 (3-d hexagonal), SBA-15 (hexagonal) and SBA-16 (cubic cage-structured) (61). This is different from MCM in that they possess larger pores of 4.6$30 \mathrm{~nm}$ and thicker silica walls. FSM-16, that is, folded sheets of mesoporous materials are another type of mesoporous materials, which are synthesized using quaternary ammonium surfactant as a template and layered polysilicate kanemite (64). Various other MSNs have been synthesized which vary in their pore symmetry and shape, TUD-1 (Technical Delft University), HMM-33 (Hiroshima Mesoporous Material-33), COK-12 (Centrum Voor Oppervlaktechemie en Katalyse/ Centre for Research Chemistry and Catalysis) (65). The schematic representation of several MSNs structures is shown in Fig. 2 as well as structural characteristics and applications of some mesoporous materials are listed in Table I.

MSNs are characterized by high surface area and large pore volume, uniform and tunable pore size, facile surface functionalization and a stable and rigid framework (71).

MSNs are commonly prepared by sol-gel "chimie douce" process in aqueous solution. This process involves the synthesis of an inorganic polymeric network at low temperature, being the gel formed from a colloidal suspension or by hydrolysis and subsequent condensation of an silica precursor $\left(\mathrm{Si}(\mathrm{OEt})_{4}\right)$, generally the most used is a tetraethyl orthosilicate, TEOS, in solution. In hydrolysis reactions, the alkoxide group (OR) of the silica precursor is replaced by the silanol group ( $\mathrm{Si}-\mathrm{OH}$ ), forming an alcohol in parallel, due to the nucleophilic attack of the oxygen atom of the water hydroxyl group $(72,73)$. In condensation reactions, dimers, linear trimers, cyclic and polymeric species with siloxane bonds (Si-O-Si) are formed (73). In order to obtain mesoporous nanomaterials, surfactants have been used, such as cetyltrimethylammonium bromide (also known as CTAB) (74). Usually, the surfactant will selfaggregate into micelles at a concentration higher than the critical micelle concentration (CMC). Then, the silica precursors can condense at the micelles surface forming MSNs. Finally, the template surfactant can be removed either by calcination or by solvent extraction to generate pores $(74,75)$, as depicted in Fig. 3 .
Considering the abundant availability of various surfactants types and the deep understanding of sol-gel chemistry, MSNs with different structures have been developed. The size, morphology, pore size, and pore structure of MSNs can be rationally designed and the synthesis process can be freely controlled.

The resulting silica-based mesoporous matrices may offer the following unique structural: 1) ordered porous structure. MSNs have along-range ordered porous structure without interconnection between individual porous channels, which allows fine control of the drug loading and release kinetics; 2) large pore volume and surface area. The pore volume and surface area of MSNs are usually above $1 \mathrm{~cm}^{3} \mathrm{~g}^{-1}$ and $700 \mathrm{~m}^{2} \mathrm{~g}^{-1}$, respectively, showing high potential for molecules loading and dissolution enhancement; 3) tunable particle size. The MSNs particle size can be controlled from 50 to $300 \mathrm{~nm}$, which is suitable for facile endocytosis by living cells; 4) two functional surfaces, namely cylindrical pore surface and exterior particle surface. These silanol-contained surfaces can be selectively functionalized to achieve better control over drug loading and release. Moreover, the external surface can be conjugated with targeting ligands for efficient cell-specific drug delivery (61,74-77).

\section{Properties Effect of MSNs in Drug Delivery Systems}

Stable suspension formation in solution, tunable pore size and structure, controlled particle size and shape, high pore volume and surface area, make the MSNs ideal for applications as nanocarriers $(12,77)$.

The pores size control influences on loading and release rates of molecules. Increasing pores size, more molecules can be loaded inside the pores and consequently, an increase of release rates can be observed $(12,77,78)$. Pores volume is an important factor on drug loading properties. High pores volume can avoid strong drug-drug interactions consequently facilitating drugpores wall intermolecular interactions leading to large filling of the mesopores channels $(12,77)$. The type of pores arrangement can also influence on cargo loading and release rates. Three distinct pore arrangement, TUD-1, MCM-41 and SBA-15 materials, were evaluated in processes on ibuprofen loading and release processes by Heikkilä et al . (79,80). TUD-1 (disordered mesoporous) materials that present 3D pores arrangement showed faster drug release than 2D hexagonal mesopores materials (SBA-15 and MCM-41). Andersson et al . (81) demonstrated that similar hexagonal mesostructured materials with interconnected (SBA1) pores displayed faster release rate than unconnected (SBA-3) pores systems. For these systems, similar drug loading amounts were detected.

Large surface area is also a crucial characteristic regarding to cargo diffusion process from MSNs. Vallet-Regí group 
Fig. 2 Schematic representation of distinct types of MSNs.

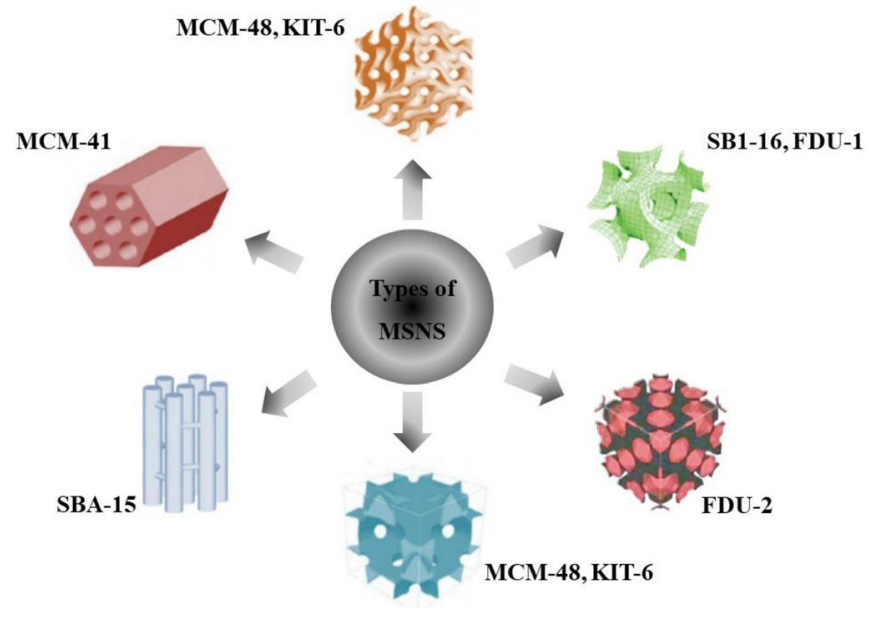

Biocompatibility and Biodistribution

82-84) reported the 2-D hexagonal mesoporous silica as carriers showing higher drugs adsorption values for MCM-41 (Specific surface area $\left(\mathrm{S}_{\mathrm{BET}}\right)=1157 \mathrm{~m}^{2} \mathrm{~g}^{-1}$ ) than for SBA$15\left(\mathrm{~S}_{\mathrm{BET}}=719 \mathrm{~m}^{2} \mathrm{~g}^{-1}\right)$ systems.

Particles size and shape can also influence their blood circulation, cellular uptake and tumor penetration, being determinant parameters to achieve therapeutic effects. Regarding particles size, a diameter range from 50 to $300 \mathrm{~nm}$ can favor an optimal cellular uptake, long circulation time, high drug loading and high accumulation in tumors (12). Huang et al . $(85,86)$ described the importance of particle shape design towards therapeutic application showing faster and higher internalization of rod-shaped MSNs on tumor cells than spherical MSNs.

\section{BIOLOGICAL BEHAVIOR OF MSNS: BIOCOMPATIBILITY, BIODISTRIBUTION, BIODEGRADABILITY AND CLEARANCE}

The biocompatibility, biodistribution, biodegradability and clearance of MSNs are subject to an ongoing discussion in research circles. In this section, conclusions are presented and a few representative studies are highlighted.
Determining the safety and biocompatibility of MSNs is crucial owing to its variable characteristics. The data generated from literature suggest that careful control of particle size and shape is the determinant factor in the biodistribution and toxicity of MSNs. In addition, the safety and toxicity of MSNs also depend on the administered MSNs dosage. Surface MSNs properties also have a great impact on their biodistribution and biocompatibility (64,87-90). The major toxicity pathway associated with silica is due to its surface chemistry (silanol groups) which can interact with the membrane components leading to the cells lysis and cellular components leaking $(91,92)$. Mesoporous silica exhibited lower hemolytic effect compared to non-porous silica (25). This could be attributed to the lower density of silanol groups on the surface of mesoporous structures $(93)$. Authors $(61,94)$ made an attempt to study the single and repeated dose MSNs toxicity following intravenous administration in mice. LD50 of MSNs was found to be higher than $1000 \mathrm{mg} \mathrm{kg}^{-1}$. In single dose toxicity studies, mice were injected with MSNs at a low dose and high dose. At the higher dose of $1280 \mathrm{mg} \mathrm{kg}^{-1}$, mice did not survive. In contrast, the groups treated with low MSNs dose did not show any behavioral changes nor any hematology or pathological changes. To carry out the detailed repeated dose toxicity studies, intravenous administration of hollow MSNs (HMSNs)
Table I Some of the Types of MSNs and Their Structural Characteristics and Applications

\begin{tabular}{llllll}
\hline MSN Family & MSN type & Pore size $(\mathrm{nm})$ & Pore symmetry & Applications & References \\
\hline M4IS & MCM-4I & $1.5-8$ & 2D hexagonal P6mm & Drug delivery & $(61,66)$ \\
& MCM-48 & $2-5$ & 3D cubic la3d & & \\
& MCM-50 & $2-5$ & Lamellar P2 & Adsorbents; Catalysis & $(67,68)$ \\
SBA & SBA-II & $2.1-3.6$ & 3D cubic Pm3m & & $(67)$ \\
& SBA-12 & 3.1 & 3D hexagonal P63/mmc & & $(61,67)$ \\
& SBA-15 & $6-0$ & 2D hexagonal p6mm & Drug delivery & $(61,69)$ \\
& SBA-16 & $5-15$ & Cubic Im3m & & $(61,70)$ \\
\hline
\end{tabular}


Fig. 3 Schematic representation of MSNs fabrication.

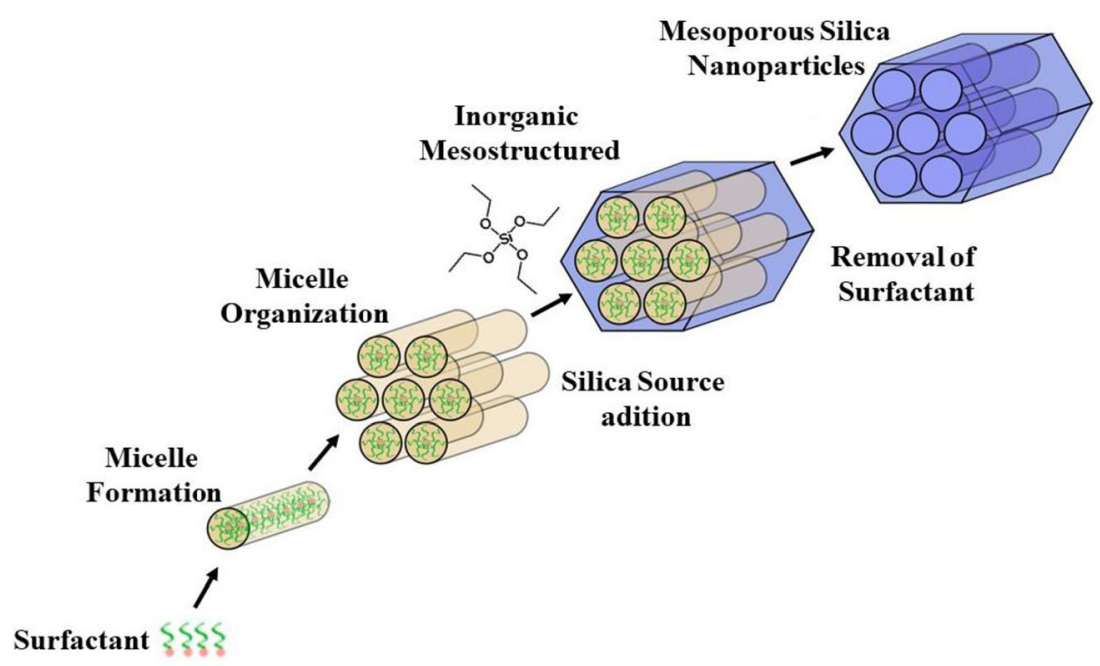

were given to mice continuously for 14 days and observed for a month. During the one-month observation period, no mortality was observed. Moreover, no remarkable changes in pathology or blood parameters were observed. In order to assess the MSNs fate after different administration routes, Fu et al . (95) tested MSNs with a particle size of $110 \mathrm{~nm}$ in ICR mice. Following administration via hypodermic, intramuscular and intravenous injection as well as oral administration, the in viwo distribution of fluorescent-tagged MSNs was tracked. It was observed that of all the exposure routes, the oral route was found to be well tolerated even when the dose was increased to $5000 \mathrm{mg} \mathrm{kg}^{-1}$ and intravenous route seemed to have the least threshold. MSNs administered via intravenous route were found to preferentially accumulate in the liver and spleen at the end of $24 \mathrm{~h}$ and 7 days whereas those administered by other routes did not show any fluorescence in these organs. It was observed that a portion of the MSNs administered via intramuscular and hypodermic routes could cross different biological barriers with a slow absorption rate. The major excretion routes of MSNs were found to be via urine and feces with the highest values after oral administration as compared to other routes. No histopathological changes were observed in liver, spleen, kidney and lung at the end of $24 \mathrm{~h}$ and 7 days by different exposure routes. The results suggested that MSNs were found to be safe and well tolerated when administered by oral and intravenous routes $(87,95)$.

\section{Biodegradability}

The silica particles do degrade into silicic acid $\left(\mathrm{Si}(\mathrm{OH})_{4}, \mathrm{pKa}\right.$ 9.6) in biological media by dissolution. Silicic acid is soluble in water and consists on the dominant silicon species at low concentration $\left(<2 \times 10^{-3} \mathrm{M}\right)(96)$. It is also excreted through the urine and its good bioavailability even contributes to maintaining bone health $(88,96)$.
The dissolution process of amorphous silica in aqueous media includes three steps: i) hydration, in which water is adsorbed into the siloxane framework, ii) hydrolysis, in which siloxane are hydrolyzed into silanols, and ii) ion exchange processes, which consist of nucleophilic attack of $\mathrm{OH}^{-}$leading to the silicic acid leaching. At the molecular level, silica hydrolyzes in aqueous media to form the same products as those of silica nanoparticles which are nontoxic and diffuse through the blood stream or the lymphatic system to be eventually cleared in the urine. Similar to silica nanoparticles, the solubility of silicic acid degradation products is key for the effective hydrolytic degradation of silica nanomaterials $(96,97)$.

Investigations have been carried out to assess the direct influence of the size, morphology and degradation medium parameters on the silica nanoparticles degradation. The physicochemical engineering of MSNs thus allows one to tune the dissolution silica rate in biorelevant media for specific biomedical applications (96). Some physicochemical parameters such as morphology, particles and pores size, surface charge and

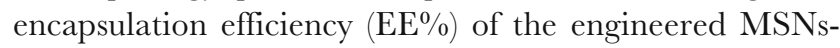
based nanocarriers for infectious diseases treatment are depicted in Table II.

\section{Effect of Size}

Chen et al . (98) demonstrated that the degradation of MSNs is independent of their diameter with 390, 310, 200, and $150 \mathrm{~nm}$ nanoparticles in simulated body fluid (SBF) at 37 ${ }^{\circ} \mathrm{C}$. During the first two days, the degradation rate was nearly $45 \%$ per day, which then slowed down to about $1 \%$ per day, and the degradation was completed in a week $(88,98,99)$. He et al . (100) investigated the role of the surface area on the degradation of mesoporous silica, comparing three samples of surface areas of 958, 829, and $282 \mathrm{~m}^{2} \mathrm{~g}^{-1}$ at fixed concentration of $0.1 \mathrm{mg} \mathrm{mL}^{-1}$ in $\mathrm{SBF}$, which were sealed in polyethylene bottles at $37^{\circ} \mathrm{C}$ and shaken at $150 \mathrm{rpm}$ with a 
Table II Physicochemical Parameters as Morphology, Particles and Pores Size, Surface Charge and Encapsulation Efficiency (EE\% of the Engineered MSNsBased Nanocarriers for Infectious Diseases Treatment

\begin{tabular}{|c|c|c|c|c|c|}
\hline Carrier/morphology & $\begin{array}{l}\text { Encapsulation } \\
\text { efficiency (\%) }\end{array}$ & Particle size (nm) & Pores size (nm) & Zeta potential (mV) & References \\
\hline $\begin{array}{l}\text { Spherical MSNs } \\
\text { respectively }\end{array}$ & $\begin{array}{l}26.8 \text { and } \\
22.5 \\
(110)\end{array}$ & respectively & $\begin{array}{l}40 \text { and } 100 \\
\text { respectively }\end{array}$ & 8 to 9 & $\begin{array}{l}-20 \text { and }- \\
16.9\end{array}$ \\
\hline Spherical MCM-4I-like MSNs & - & 150 & - & -25 & $(|| \mid)$ \\
\hline $\begin{array}{l}\text { Spherical MSNs-MCM-4I, modified (vi- } \\
\text { nyl groups) MSNs-MCM-4I and } \\
\text { spherical MSNs-MCM-48 }\end{array}$ & 20 & $\begin{array}{l}\text { Modified and non-modified } \\
\text { MSNs-MCM-4I: } \sim 60 \text { and } \\
\text { MSNs-MCM-48: } \sim 46\end{array}$ & $\begin{array}{l}\text { MCM-4I types: } 2.5- \\
3.1 \text { and MCM-48 } \\
\text { type: } 2.5-9\end{array}$ & - & $(\mid 12)$ \\
\hline Spherical MSNs & - & 80 & - & -26.7 & $(1 \mid 3)$ \\
\hline Spherical MSNs & - & 210 & 2.4 & - & $(|| 4)$ \\
\hline $\begin{array}{l}\text { Rod shaped HMSNs with large cone- } \\
\text { shaped pores and small cone-shaped } \\
\text { pores }\end{array}$ & 35 and 5 & respectively & $\begin{array}{l}\text { Length and width of } \\
400 \text { and } 200 \text {, re- } \\
\text { spectively, for } \\
\text { both } \\
\text { nanosystems }\end{array}$ & 40 and 3, respectively & - \\
\hline Spherical MSNs-MCM-4I & - & $200-400$ & 1.75 to 2.98 & - & $(116)$ \\
\hline $\begin{array}{l}\text { MSNs-SBA-16 and MSMs-SBA- I 6/hy- } \\
\text { droxyapatite (HA) } \\
\text { respectively }\end{array}$ & $\begin{array}{l}28 \text { to } 31 \\
(117)\end{array}$ & respectively & - & 3.4 and 3.8 , respectively & $\begin{array}{l}-56 \text { and } \\
2.9\end{array}$ \\
\hline Spherical MSNs & 5.9 to 26.8 & 100 & 2.8 & -21.2 & $(1 \mid 8)$ \\
\hline Spherical and spherical modified MSNs & 7.8 & $\sim 150$ & 2.4 & $\begin{array}{l}-36.4 \text { for non-modified MSNs } \\
\text { and }+31.8 \text { to }+37.4 \text { for } \\
\text { modified MSNs }\end{array}$ & $(119)$ \\
\hline $\begin{array}{l}\text { Spherical MSNs-supported Ag-Bi } \\
\text { nanoparticles }\end{array}$ & - & $200-240$ & 3.4-4.8 & - & $(120)$ \\
\hline $\begin{array}{l}\text { Spherical MSNs with large cone-shaped } \\
\text { pores }\end{array}$ & $\begin{array}{l}\text { Ag: } 2 \text { and } \\
\text { DNase I: } \\
32\end{array}$ & 200 & $42-43$ & - & $(|2|)$ \\
\hline Spherical modified magnetic MSNs & 10.7 & 240 & 3 & -5.32 & $(122)$ \\
\hline zSpherical MSNs-MCM-4I & - & $150-300$ & 2 & $\begin{array}{l}\text { MSNs: }-45 \text { and MSNs-Ag: } \\
\quad+48\end{array}$ & $(123)$ \\
\hline $\begin{array}{l}\text { Spherical MSNs-MCM-4I and spherical } \\
\text { modified MSNs-MCM-4I }\end{array}$ & - & $122-220$ & 1.9-2.4 & $\begin{array}{l}\text { MSNs: }-30 \text { and modified } \\
\text { MSNs: }+5 \text { to }+25\end{array}$ & $(124)$ \\
\hline $\begin{array}{l}\text { Spherical MSNs-MCM-4I and spherical } \\
\text { modified MSNs-MCM-4I }\end{array}$ & 36 & 182 & - & -30 & $(125)$ \\
\hline $\begin{array}{l}\text { Spherical MSNs and spherical modified } \\
\text { MSNs }\end{array}$ & I I to 40 & $\sim 72$ & $3.3-4$ & $\begin{array}{l}\text { MSNs: }-22.9 \text { and modified } \\
\text { MSNs: } \\
\text { Aminated MSNs: }+19 \\
\text { Carboxyl functionalized MSNs: } \\
\quad-53\end{array}$ & $(126)$ \\
\hline SBA- 15 and modified SBA- 15 & $93-98$ & $100-300$ & $\begin{array}{l}\text { SBA- I } 5: 7 \\
\text { And modified SBA- } \\
\quad \text { I } 5: 6-6.2\end{array}$ & - & $(127)$ \\
\hline $\begin{array}{l}\text { Spherical MSNs-MCM-48 and spherical } \\
\text { modified MSNs-MCM-48 }\end{array}$ & $27-31.5$ & | 50-600 & 3.2 & $\begin{array}{l}\text { MSNs-MCM-48: }-28 \text { to }-34 \\
\text { and modified MSNs-MCM- } \\
\text { 48: }+30 \text { to }+63\end{array}$ & $(128)$ \\
\hline $\begin{array}{l}\text { modified porous silicon nanoparticles } \\
\text { (UnTHCPSi NPs) }\end{array}$ & - & $176-184$ & - & -25.5 & $(\mid 29)$ \\
\hline $\begin{array}{l}\text { Spherical MSNs-MCM-4I and spherical } \\
\text { modified MSNs-MCM-4I }\end{array}$ & - & 90 & - & - & $(130)$ \\
\hline $\begin{array}{l}\text { Hollow oblate MSNs and modified } \\
\text { hollow oblate MSNs }\end{array}$ & $56-64$ & $\begin{array}{l}\text { Average long-axis length of } \\
\sim 200 \text { and a short-axis width } \\
\text { of } \sim 100\end{array}$ & $3.3-3.7$ & - & $(|3|)$ \\
\hline $\begin{array}{l}\text { Spherical MSNs-MCM-4I and spherical } \\
\text { modified MSNs-MCM-4I }\end{array}$ & - & $\sim 50$ and $\sim 100$ & $\sim 2$ & $\begin{array}{l}\text { MSNs-MCM-4I: }-26 \text { to }-29 \\
\text { and modified MSNs-MCM- } \\
41:+38 \text { to }+42.5\end{array}$ & $(132)$ \\
\hline & - & $100-130$ & 2.5 & & $(133)$ \\
\hline
\end{tabular}


Table II (continued)

\begin{tabular}{|c|c|c|c|c|c|}
\hline Carrier/morphology & $\begin{array}{l}\text { Encapsulation } \\
\text { efficiency (\%) }\end{array}$ & Particle size (nm) & Pores size (nm) & Zeta potential $(\mathrm{mV})$ & References \\
\hline $\begin{array}{l}\text { Spherical MSNs and spherical modified } \\
\text { MSNs }\end{array}$ & & & & $\begin{array}{l}\text { MSNs: }-7 \text { to }-10.5 \text { and modi- } \\
\text { fied MSNs: }+30 \text { to }+39\end{array}$ & \\
\hline $\begin{array}{l}\text { Spherical MSNs-MCM-4I and spherical } \\
\text { modified MSNs-MCM-4I }\end{array}$ & Up to $\sim 70$ & 100 & $\sim 2$ & $\begin{array}{l}\text { Phosphonate silane-modified } \\
\text { MCM-4I: }-46.3 \text { and amine- } \\
\text { silane modified MCM-4I: } \\
38.8\end{array}$ & $(134)$ \\
\hline Spherical modified MSNs & - & 117 & 3.8 & +36 & $(135)$ \\
\hline Spherical MSNs & Up to $\sim 35$ & 38.8 & $\sim 7$ & -33 & $(136)$ \\
\hline $\begin{array}{l}\text { Spherical MSNs-MCM-4I and spherical } \\
\text { modified MSNs-MCM-4I }\end{array}$ & - & 110 & 3.3 & $\begin{array}{l}\text { MSNs-MCM-4I: }-21 \text { and } \\
\text { modified MSNs-MCM-4I: } \\
+45.5\end{array}$ & $(137)$ \\
\hline Spherical MSNs & 83 & 100 to 105 & - & +32 to +30 & $(138)$ \\
\hline $\begin{array}{l}\text { Mesoporous silica whiskers-SBA- I } 5 \text { and } \\
\text { modified mesoporous silica whiskers- } \\
\text { SBA- I } 5\end{array}$ & 62.6 to 71.4 & 50 & 6 & - & $(139)$ \\
\hline $\begin{array}{l}\text { Spherical MSNs-MCM-4I and spherical } \\
\text { modified MSNs-MCM-4I }\end{array}$ & $\begin{array}{l}\text { Up to } 42 \text { and } \\
63 \%\end{array}$ & $39-91$ & 2.5 & $\begin{array}{l}\text { MSNs-MCM-4I: }-12 \text { and } \\
\text { modified MSNs-MCM-4I: } \\
+32.5 \text { to }+33.6\end{array}$ & $(140)$ \\
\hline $\begin{array}{l}\text { Spherical MSNs and spherical modified } \\
\text { MSNs }\end{array}$ & 58.5 & 500 & 2 & - & $(|4|)$ \\
\hline Spherical MSNs-MCM-4I & - & 100 & $3-3.5$ & - & $(\mid 42)$ \\
\hline Spherical MSNs & 10.7 & $20-40$ & 2.5 to 3.1 & - & $(143)$ \\
\hline Spherical MSNs & - & 400 & $\sim 7.6$ & - & $(144)$ \\
\hline $\begin{array}{l}\text { Spherical MSNs and spherical modified } \\
\text { MSNs }\end{array}$ & 2 & $75-88$ & $2.6-3.5$ & $\begin{array}{l}\text { MSNs: }-25 \text { and modified } \\
\text { MSNs: }-0.26\end{array}$ & $(\mid 45)$ \\
\hline $\begin{array}{l}\text { Spherical MSNs and spherical modified } \\
\text { MSNs }\end{array}$ & - & I 50-200 & 2 & $\begin{array}{l}\text { MSNs: }-39.6 \text { and modified } \\
\text { MSNs: }-29.5\end{array}$ & $(146)$ \\
\hline $\begin{array}{l}\text { Spherical MSNs and spherical modified } \\
\text { MSNs }\end{array}$ & $30-34$ & $100-150$ & 2 & $\begin{array}{l}\text { MSNs: }-28.8 \text { to }-44.1 \text { and } \\
\text { modified MSNs: }-32 \text { to }-40\end{array}$ & $(147)$ \\
\hline $\begin{array}{l}\text { Spherical MSNs and spherical modified } \\
\text { MSNs }\end{array}$ & - & $354-57 \mid$ & - & -11.2 to -20.5 (in PBS) & $(54)$ \\
\hline Spherical HMSNs & 15 & 200 & - & - & $(\mid 48)$ \\
\hline
\end{tabular}

mechanical shaker. This study indicated, on the one hand, that there was a burst degradation in the first 2 to $4 \mathrm{~h}$, leading to 30,70 , and $90 \%$ of silica hydrolytic degradation as the surface increased, and, on the other hand, a complete degradation was obtained in 15 days.

\section{Effects of Morphology and Degradation Medium}

A remarkable study was reported by Li et al . (101) regarding the degradation of MSNs with spherical and rod-shaped morphologies with aspect ratios (ARs) of 1.75 and 5. Authors investigated these nanoparticles by TEM and sample weight measurements after degradation for 7 days by soaking in three different degradation media: simulated gastric fluid (SGF, pH 1.2), simulated intestinal fluid (SIF, pH 6.5), and SBF ( $\mathrm{pH} 7$ ). After a week in acidic gastric fluid, none of the three MSNs did displayed significant alteration, and their degradation was only about $10 \mathrm{wt} \%$ for all nanoparticles. On the contrary, the degradation was much more pronounced and AR dependent in both simulated intestinal and body fluids. In general, the
SIF generated more degradation than the SBF. The same group also showed that spherical MSNs were more rapidly degraded than rod-like MSNs with ARs of 2 and 4, and the presence of FBS in the Dulbecco's modified Eagle medium (DMEM) accelerated the degradation process $(88,102)$.

\section{Clearance}

When ingested by human beings through food sources, silicon species circulate through the blood plasma and are absorbed in the form of silicic acid being $41 \%$ excreted in the urine (103). In the case of silica-based nanoparticles, the particle dissolution into silicic acid and its subsequent excretion was demonstrated in vivo on mice models as well as in human clinical trials $(88,103)$. It is challenging to confidently draw trends between separated studies describing the clearance of silica nanoparticles because of the plethora of varying parameters, not least of which are the nanoparticle characteristics. Nevertheless, on animal models, several trends have been observed between MSNs characteristics and their clearance: i) 
the effect of size, ii) the effect of surface functions, iii) the effect of surface charge, and iv) the effect of morphology.

\section{Effect of Size}

He et al . (104) reported an in-depth study of bare MSNs of various diameters (80, 120, 200, and $360 \mathrm{~nm})$ and observed a significant nanoparticles excretion from 15 to $45 \%$ after the first 30 min with consistently higher excretion percentages for larger nanoparticles. Burns et al . (105) have shown that nonporous silica quantum dots (3-6 nm) can be effectively $(\approx 70 \%)$ cleared out of an animal body by renal excretion in 2 days, while Kumar et al . (106) showed that larger silica nanoparticles $(20-25 \mathrm{~nm})$ preferred hepatobiliary excretion, with a complete clearance over a period of 15 days. Cho et al . (107) studied dye-labeled nonporous silica nanoparticles of 50, 100 and $200 \mathrm{~nm}$ and demonstrated their clearance in the urine and bile. The $50 \mathrm{~nm}$ nanoparticles cleared faster than 100 and $200 \mathrm{~nm}$ ones, and $200 \mathrm{~nm}$ nanoparticles were less excreted.

\section{Effect of Surface Functions}

Some authors $(96,104,108)$ showed that PEG-modified $45 \mathrm{~nm}$ nonporous silica nanoparticles exhibit a much longer blood circulation time $\left(t^{1 / 2}=180 \pm 40 \mathrm{~min}\right)$ than unmodified silica nanoparticles $\left(t^{1} / 2=80 \pm 30 \mathrm{~min}\right)$ and carboxylated silica nanoparticles $\left(t^{1 / 2}=35 \pm 10 \mathrm{~min}\right)$, being partly excreted via renal clearance. A stealth behavior is acquired by PEGylation, which reduces the RES uptake and increases the circulation half-life. As a result, PEGylated MSNs of various sizes (80, 120,200 , and $360 \mathrm{~nm}$ ) were excreted slower than unfunctionalized ones due to the much slower particle capture by the liver and spleen.

\section{Effect of Charge}

The particle charge-clearance dependence studies indicated that MSNs with a positive charge $(+34 \mathrm{mV})$ at neutral $\mathrm{pH}$ are rapidly excreted from the liver into the gastrointestinal tract and then eliminated through the feces, while negatively charged $(-18 \mathrm{mV})$ MSNs remained trapped in the liver $(104,109)$.

\section{Effect of Morphology}

The morphology influence on the clearance displayed that mesoporous silica nanorods of aspect ratio $\approx 1.5$ (185 \pm $22 \mathrm{~nm}$ long) are more rapidly cleared than longer nanorods of aspect ratio around $5(720 \pm 65 \mathrm{~nm}$ long). Comparing spherical-shaped and rod-shaped MSNs, nanospheres were mostly excreted during the first few hours, while nanorods had slower clearance rates $(88,99)$. Several research groups consistently found nearly intact MSNs in the mice urine. Rod-shaped MSNs of various aspect ratios were also found in the urine and feces of mice (96).

\section{MSNS FOR INFECTIOUS DISEASES TREATMENT}

The direct drugs application in preclinical trials can generate undesirable changes in their physicochemical, pharmacokinetic and biological properties, minimizing their action in infectious diseases treatment associated with the risk of resistance by pathogens. In this context, the development of smart and specific MSNs-based nanocarriers comprise a promising and effective approach for efficient drug delivery and consequently infectious diseases treatment $(149,150)$. Table III depicted several examples of MSNs for infectious diseases treatment including bacterial, parasitic, fungal and viral infections as well as drugs type, MSNs-based carriers, microorganisms and drugs absorption and release mechanisms.

\section{Bacterial Infections}

The great challenge concerning bacterial infections treatment comprises the development of antibiotic resistance in several bacteria species, leading to ineffective treatment. One way to overcome this drawback consists on to use nanoparticles that can interact efficiently to the bacterial surface disrupting their cell wall leading to cell death. Interestingly, multifunctional nanoparticles can also interact with cellular organelles and biomolecules present in the bacteria, making difficult the resistance development against them $(1,151)$.

Considering the abovementioned advantages, authors have reported the use of MSNs containing peptides with antibacterial properties, aiming to prevent their enzymatic degradation potentiating their antimicrobial activity (110). It can also guarantee the same effect for loading antimicrobial proteins (115). Other studies reported the use of hydrophobic antimicrobials drugs-loaded MSNs to enhance their effect in biological media $(112,113,118)$. On the other hand, for hydrophilic antimicrobial drugs which are very soluble in an aqueous/biological environments, MSNs can also promote a controlled drug release (116).

The association of multifunctional MSNs with antimicrobial drugs allows besides controlled drug release, efficient internalization in the bacterial environment (111,152). Drugloaded mesoporous silica carriers were reported to access bone infections since fluids circulation in this region is very low which reduce drugs bioavailability via conventional administration $(114,117)$. In addition, the MSNs specificity can be achieved by easily functionalization improving nanoplatforms action in target sites and controlled drugs release minimizing side effects. Table III summarized examples of MSNs 
Table III MSNs as Drug Delivery Platforms for Infectious Diseases Treatment Including Bacterial, Parasitic, Fungal and Viral Infections Besides Drugs Type, MSNs-Based Carriers, Microorganisms and Drugs Absorption and Release Mechanisms

\begin{tabular}{|c|c|c|c|c|c|c|}
\hline Drug & Carrier & Assay & Microorganism & $\begin{array}{l}\text { Adsorption } \\
\text { mechanism }\end{array}$ & $\begin{array}{l}\text { Release } \\
\text { mechanism }\end{array}$ & References \\
\hline Rifampicin (RIF) & MSNs with 40 and $100 \mathrm{~nm}$ & In vitro & Staphylococcus aureus & $\begin{array}{l}\text { Passive diffusion } \\
\text { method }\end{array}$ & Diffusion & $(1 \mid 0)$ \\
\hline $\begin{array}{l}\text { Levofloxacin } \\
\quad(\text { LEVO) }\end{array}$ & $\begin{array}{l}\text { MSNs decorated with lectin concanavalin A (ConA) } \\
\left(\mathrm{MSN}_{\text {ConA }}\right)\end{array}$ & In vitro & Escherichia coli & $\begin{array}{l}\text { Impregnation } \\
\text { method }\end{array}$ & Diffusion & $(|| \mid)$ \\
\hline $\begin{array}{l}\text { Norfloxacin } \\
\text { (NFX) }\end{array}$ & $\begin{array}{l}\text { MSNs-MCM-4I, modified (vinyl groups) MSNs- } \\
\text { MCM-4I and MSNs-MCM-48 }\end{array}$ & In vitro & E. coli & $\begin{array}{l}\text { Impregnation } \\
\text { method }\end{array}$ & $\begin{array}{l}\text { Dissociation/ } \\
\text { diffusion }\end{array}$ & $(1 \mid 2)$ \\
\hline $\begin{array}{l}\text { Gentamicin } \\
\quad(\text { GEN })\end{array}$ & $\begin{array}{l}\text { MSNs modified with a lipid bilayer surface shell and a } \\
\text { bacterial-targeting peptide Ubiquicidin }\left(\mathrm{UB}_{29-41}\right) \\
\text { (MSNs-LU) }\end{array}$ & $\begin{array}{l}\text { In vitro and } \\
\text { in vivo } \\
\text { (S. aureus- } \\
\text { infected } \\
\text { mice) }\end{array}$ & S. aureus & $\begin{array}{l}\text { Impregnation } \\
\text { method }\end{array}$ & $\begin{array}{l}\text { Bacteria-re- } \\
\quad \text { sponsive } \\
\text { controlled } \\
\text { release }\end{array}$ & $(1 \mid 3)$ \\
\hline $\begin{array}{l}\text { Vancomycin } \\
\text { (VAN) }\end{array}$ & MSNs dispersed onto gelatin matrix & $\begin{array}{l}\text { In vitro and } \\
\text { in vivo (in- } \\
\text { duced } \\
\text { bone in- } \\
\text { fection) }\end{array}$ & S. aureus & $\begin{array}{l}\text { Impregnation } \\
\text { method }\end{array}$ & $\begin{array}{l}\text { Diffusion/sus- } \\
\text { tained } \\
\text { release }\end{array}$ & $(1 \mid 4)$ \\
\hline $\begin{array}{l}\text { Lysozyme } \\
\text { (LYZ) }\end{array}$ & $\begin{array}{l}\text { Hollow MSNs (HMSNs) with large and small cone } \\
\text { shaped pores (HMSN-LP and HMSN-SP, } \\
\text { respectively) }\end{array}$ & In vitro & E. coli & $\begin{array}{l}\text { Ultrasonication } \\
\text { method }\end{array}$ & $\begin{array}{l}\text { Diffusion/sus- } \\
\text { tained } \\
\text { release }\end{array}$ & $(115)$ \\
\hline $\begin{array}{l}\text { Doxycycline } \\
\text { (DOXY) }\end{array}$ & $\begin{array}{l}\text { MCM- } 4 \text { I modified with phenyl, mercaptopropyl, } \\
\text { propylsulfonic and magnesium }\left(\mathrm{MCM}-\mathrm{C}_{6} \mathrm{H}_{5}\right. \\
\mathrm{MCM}-\mathrm{SH}, \mathrm{MCM}-\mathrm{SO}_{3} \mathrm{H} \text { and } \mathrm{MCM}-\mathrm{Mg} \text {, } \\
\text { respectively) }\end{array}$ & In vitro & Klebsiella pneumoniae & $\begin{array}{l}\text { Impregnation } \\
\text { method }\end{array}$ & $\begin{array}{l}\text { Dissociation/ } \\
\text { diffusion }\end{array}$ & $(116)$ \\
\hline $\begin{array}{l}\text { Ciprofloxacin } \\
\quad(\text { CIP) }\end{array}$ & $\begin{array}{l}\text { MSNs-SBA-16/hydroxyapatite (HA) modified with 3- } \\
\text { aminopropyltriethoxysilane (APTES), anchored } \\
\text { with diethylenetriaminepentaacetic acid (DTPA) for } \\
\text { chelating technetium-99m ( }{ }^{99} \text { mTc-DTPA-SBA- } 16- \\
\text { APTES) }\end{array}$ & In vitro & $\begin{array}{l}\text { S. aureus, } P \text {. aeruginosa, } \\
\text { E. coli and Bacillus cereus }\end{array}$ & $\begin{array}{l}\text { Impregnation } \\
\text { method }\end{array}$ & $\begin{array}{l}\text { Diffusion/sus- } \\
\text { tained } \\
\text { release }\end{array}$ & $(117)$ \\
\hline $\begin{array}{l}\text { Clofazimine } \\
\quad(\text { CFZ) }\end{array}$ & MSNs loaded with CFZ + acetophenone (AP) & In vitro & $\begin{array}{l}\text { Mycobacterium } \\
\text { tuberculosis }\end{array}$ & $\begin{array}{l}\text { Impregnation } \\
\text { method }\end{array}$ & $\begin{array}{l}\text { Diffusion/AP- } \\
\text { assisted } \\
\text { release }\end{array}$ & $(1 \mid 8)$ \\
\hline $\begin{array}{l}\text { Levofloxacin } \\
\quad(\text { LEVO) }\end{array}$ & $\begin{array}{l}\text { MSN modified with [3-(2-aminoethylamino) propyl]- } \\
\text { trimethoxysilane (DAMO) (MSNs-DAMO) }\end{array}$ & In vitro & E. coli & $\begin{array}{l}\text { Impregnation } \\
\text { method }\end{array}$ & $\begin{array}{l}\text { Diffusion/sus- } \\
\text { tained } \\
\text { release }\end{array}$ & $(1 \mid 9)$ \\
\hline \multirow{2}{*}{$\begin{array}{l}\text { Silver and bis- } \\
\text { muth nano- } \\
\text { particles (Ag } \\
\text { and } \mathrm{Bi} \text {, } \\
\text { respectively) }\end{array}$} & Ag-Bi@MSNs & $\begin{array}{l}\text { In vitro and } \\
\text { in vivo }\end{array}$ & $\begin{array}{l}\text { methicillin-resistant } \\
\text { S. Aureus (MRSA) }\end{array}$ & $\begin{array}{l}\text { In situ growth of } \\
\mathrm{Ag} \text { and } \mathrm{Bi}\end{array}$ & & \\
\hline & nanoparticles & $\begin{array}{l}\text { Near-infra- } \\
\quad \text { red } \\
\text { (NIR)-re- } \\
\text { sponsive } \\
\text { con- } \\
\text { trolled } \\
\text { release }\end{array}$ & $(120)$ & & & \\
\hline $\begin{array}{l}\text { DNase } \\
\text { I(Deoxyrib- } \\
\text { ose nucle- } \\
\text { ase I) }\end{array}$ & MSN-Ag & In vitro & $\begin{array}{l}\text { E. coli and Streptococcus } \\
\text { mutans biofilms }\end{array}$ & $\begin{array}{l}\text { Impregnation } \\
\text { method }\end{array}$ & $\begin{array}{l}\text { Diffusion/sus- } \\
\text { tained } \\
\text { release }\end{array}$ & $(|2|)$ \\
\hline $\begin{array}{l}\text { Vancomycin } \\
\text { (VAN) }\end{array}$ & $\begin{array}{l}\text { sufonated-hyaluronic acid (S-HA) terminated mag- } \\
\text { netic MSNs modified with S. aureus antibody (Ab) } \\
\text { (Ab@S-HA@MMSNs) }\end{array}$ & In vitro & S. aureus & $\begin{array}{l}\text { Impregnation } \\
\text { method }\end{array}$ & $\begin{array}{l}\text { Hyaluronidase } \\
\text { (Hyal)-re- } \\
\text { sponsive } \\
\text { controlled } \\
\text { release }\end{array}$ & $(122)$ \\
\hline $\begin{array}{l}\text { Silver nanopar- } \\
\quad \text { ticles }(\mathrm{Ag})\end{array}$ & $\begin{array}{l}\text { poly-L-glutamic acid (PG) and polyallylamine hydro- } \\
\text { chloride (PAH) assembled by the layer-by-layer } \\
\text { (LBL) technique onto MSN-Ag (LBL@MSN-Ag) } \\
\text { nanoparticles }\end{array}$ & $\begin{array}{l}\text { In vitro and } \\
\text { in vivo } \\
\text { v8 enzyme- }\end{array}$ & $\begin{array}{l}\text { S. aureus-associated os- } \\
\text { teomyelitis infections } \\
\text { responsive controlled } \\
\text { release }\end{array}$ & $\begin{array}{l}\text { In situ growth of } \\
\mathrm{Ag} \\
(123)\end{array}$ & & \\
\hline
\end{tabular}


Table III (continued)

\begin{tabular}{|c|c|c|c|c|c|c|}
\hline Drug & Carrier & Assay & Microorganism & $\begin{array}{l}\text { Adsorption } \\
\text { mechanism }\end{array}$ & $\begin{array}{l}\text { Release } \\
\text { mechanism }\end{array}$ & References \\
\hline $\begin{array}{l}\text { Levofloxacin } \\
\qquad(\text { LEVO) }\end{array}$ & $\begin{array}{l}\text { mixed-charge pseudo-zwitterionic MSNs (ZMSN) } \\
\text { and PEGylated (PEG)-MSN }\end{array}$ & In vitro & E. coli and S. aureus & $\begin{array}{l}\text { Impregnation } \\
\text { method }\end{array}$ & $\begin{array}{l}\text { Diffusion/sus- } \\
\text { tained } \\
\text { release }\end{array}$ & $(124)$ \\
\hline $\begin{array}{l}\text { Moxifloxacin } \\
\qquad(\mathrm{MXF})\end{array}$ & disulfide snap-top redox-operated MSNs (MSN-SS) & In vivo & Francisella tularensis & $\begin{array}{l}\text { Impregnation } \\
\text { method }\end{array}$ & $\begin{array}{l}\text { Redox-respon- } \\
\text { sive con- } \\
\text { trolled } \\
\text { release }\end{array}$ & $(125)$ \\
\hline Polymyxin B & $\begin{array}{l}\text { MSNs, aminated MSNs (N-MSNs) and carboxyl } \\
\text { modified MSNs (C-MSNs) }\end{array}$ & In vitro & $\begin{array}{l}\text { P. aeruginosa, E. coli and } \\
\text { Acinetobacter } \\
\text { baumannii }\end{array}$ & $\begin{array}{l}\text { Impregnation } \\
\text { method }\end{array}$ & $\begin{array}{l}\text { Diffusion/sus- } \\
\text { tained } \\
\text { release }\end{array}$ & $(126)$ \\
\hline $\begin{array}{l}\text { Levofloxacin } \\
\text { (LEVO) }\end{array}$ & $\begin{array}{l}\text { MSNs-like SBAI 5, SBAI5@ } \mathrm{NH}_{2} \text { and } \\
\text { SBAI5@NH } / \text { PLA(polylactic acid) }\end{array}$ & In vitro & $\begin{array}{l}\text { S. aureus, E. coli, C. albi- } \\
\text { cans and Aspergillus } \\
\text { niger }\end{array}$ & $\begin{array}{l}\text { Impregnation } \\
\text { method }\end{array}$ & $\begin{array}{l}\text { Acid } \mathrm{pH}- \\
\text { controlled } \\
\text { release }\end{array}$ & $(127)$ \\
\hline $\begin{array}{l}\text { Biocide } \\
\text { Parmetol } \\
\text { SI } 5\end{array}$ & $\begin{array}{l}\text { MSNs-like MCM-48 modified with quaternary am- } \\
\text { monium salts (QASs) }\end{array}$ & In vitro & S. aureus and E. coli & $\begin{array}{l}\text { Impregnation } \\
\text { method }\end{array}$ & $\begin{array}{l}\text { Diffusion/sus- } \\
\text { tained } \\
\text { release }\end{array}$ & $(128)$ \\
\hline $\begin{array}{l}\text { Ethionamide } \\
\qquad(\mathrm{ETH})\end{array}$ & $\begin{array}{l}\text { carboxylic acid functionalized thermally hydrocarbon- } \\
\text { ized porous silicon nanoparticles (UnTHCPSi NPs) }\end{array}$ & In vitro & $\begin{array}{l}\text { multidrug-resistant } \\
\text { M. tuberculosis } \\
\text { (MRD-TB) }\end{array}$ & $\begin{array}{l}\text { Covalent con- } \\
\text { jugation of } \\
\text { ETH }\end{array}$ & - & $(129)$ \\
\hline $\begin{array}{l}\text { Moxifloxacin } \\
\qquad(\mathrm{MXF})\end{array}$ & $\begin{array}{l}\text { disulfide snap-top redox-operated MSNs modified } \\
\text { with } \beta \text {-cyclodextrin }(\beta-C D) \text { (MSN-SS- } \beta-C D)\end{array}$ & $\begin{array}{l}\text { In vitro and } \\
\text { in vivo }\end{array}$ & Francisella tularensis & $\begin{array}{l}\text { Impregnation } \\
\text { method }\end{array}$ & $\begin{array}{l}\text { Redox-respon- } \\
\text { sive con- } \\
\text { trolled } \\
\text { release }\end{array}$ & $(130)$ \\
\hline Isoniazid (INH) & $\begin{array}{l}\text { Hollow oblate MSNs (HOMSNs), trehalose- } \\
\text { conjugated HOMSNs (HOMSNs-Tre) and } \\
\text { mannose-conjugated HOMSNs (HOMSNs-Man) }\end{array}$ & In vitro & $\begin{array}{l}\text { M. smegmatis (INH-re- } \\
\text { sistant), } \\
\text { Staphylococcus } \\
\text { epidermidis and E. coli }\end{array}$ & $\begin{array}{l}\text { Impregnation } \\
\text { method }\end{array}$ & $\begin{array}{l}\text { Slow/sustained } \\
\text { release }\end{array}$ & $(|3|)$ \\
\hline Isoniazid (INH) & $\begin{array}{l}\text { MSNs-CHO (aldehyde modified)-INH and MSNs- } \\
\mathrm{CHO}-\mathrm{INH} \text { - poly(ethylene imine)-poly(ethylene } \\
\text { glycol) (PEI-PEG) }\end{array}$ & $\begin{array}{l}\text { In vitro and } \\
\text { in vivo }\end{array}$ & M. tuberculosis & $\begin{array}{l}\text { Impregnation } \\
\text { method }\end{array}$ & $\begin{array}{l}\mathrm{pH} \text {-responsive } \\
\text { controlled } \\
\text { release }\end{array}$ & $(132)$ \\
\hline $\begin{array}{l}\text { Isoniazid (INH) } \\
\text { and rifampin } \\
\text { (RIF) }\end{array}$ & $\begin{array}{l}\text { MSNs, PEI-MSNs and MSNs-MBI (I-methyl- I H- } \\
\text { benzimidazole)- } \beta C D \text { (beta-cyclodextrin) }\end{array}$ & $\begin{array}{l}\text { In vitro and } \\
\text { in vivo }\end{array}$ & M. tuberculosis & $\begin{array}{l}\text { Impregnation } \\
\text { method }\end{array}$ & $\begin{array}{l}\text { pH-responsive } \\
\text { controlled } \\
\text { release }\end{array}$ & $(133)$ \\
\hline $\begin{array}{l}\text { Moxifloxacin } \\
\text { (MFX) }\end{array}$ & $\begin{array}{l}\text { MSNs-ANA (anilinoalkane)- } \alpha C D \text { (alpha-cyclodextrin) } \\
\text { and MSNs-MBI ( I-methyl- I H-benzimidazole)- } \\
\text { BCD (beta-cyclodextrin) }\end{array}$ & $\begin{array}{l}\text { In vitro and } \\
\text { in vivo }\end{array}$ & F. tularensis & $\begin{array}{l}\text { Impregnation } \\
\text { method }\end{array}$ & $\begin{array}{l}\text { pH-responsive } \\
\text { controlled } \\
\text { release }\end{array}$ & $(134)$ \\
\hline $\begin{array}{l}\text { Model drugs } \\
\text { (Fluorescein } \\
\text { and } \\
\text { Hoechst } \\
\text { 33342) }\end{array}$ & MSNs- Ft-LVS-LPS-FBII & In vitro & F. tularensis & $\begin{array}{l}\text { Impregnation } \\
\text { method }\end{array}$ & $\begin{array}{l}\text { Pathogen (Ft- } \\
\text { LVS-LPS)- } \\
\text { responsive } \\
\text { controlled } \\
\text { release }\end{array}$ & $(135)$ \\
\hline $\begin{array}{l}\text { Antigenic } \\
\text { Preparation } \\
\text { of Soluble } \\
\text { Worm } \\
\text { (SWAP) }\end{array}$ & MSNs & In vivo & S. mansoni & $\begin{array}{l}\text { Impregnation } \\
\text { and sonica- } \\
\text { tion } \\
\text { methods }\end{array}$ & - & $(136)$ \\
\hline $\begin{array}{l}\text { Benznidazole } \\
\quad(B Z)\end{array}$ & $\begin{array}{l}\text { MSNs-MCM-4I functionalized with (3- } \\
\text { glycidoxypropy) trimethoxysilane (GPTMS) and } \\
\text { chitosan succinate (CS) }\end{array}$ & In vitro & T. cruzi & $\begin{array}{l}\text { Simple impreg- } \\
\text { nation } \\
\text { Method }\end{array}$ & - & $(137)$ \\
\hline $\begin{array}{l}\text { Praziquantel } \\
\qquad(P Z Q)\end{array}$ & MSNs & In vivo & S. mansoni & $\begin{array}{l}\text { Simple impreg- } \\
\text { nation } \\
\text { method }\end{array}$ & $\begin{array}{l}\text { Sustained } \\
\text { release }\end{array}$ & $(138)$ \\
\hline $\begin{array}{l}\text { Metronidazole } \\
\qquad(\mathrm{MNZ})\end{array}$ & $\begin{array}{l}\text { Mesoporous silica whiskers-SBA- } 15 \text { modified with } \\
\text { tannic acid (TA) }\end{array}$ & In vitro & Trichomonas gallinae & $\begin{array}{l}\text { Simple } \\
\text { Impregnatio- } \\
\text { n method }\end{array}$ & $\begin{array}{l}\text { pH-responsive } \\
\text { controlled } \\
\text { release }\end{array}$ & $(139)$ \\
\hline $\begin{array}{l}\text { Econazole } \\
\text { (ECO) }\end{array}$ & $\begin{array}{l}\text { MSNs functionalized with aminopropyl groups } \\
\qquad\left(\mathrm{MCM}-4 \mathrm{I}-\mathrm{NH}_{2}\right)\end{array}$ & $\begin{array}{l}\text { In vitro and } \\
\text { In vivo }\end{array}$ & C. albicans & $\begin{array}{l}\text { Simple impreg- } \\
\text { nation } \\
\text { method }\end{array}$ & $\begin{array}{l}\text { Slow/sustained } \\
\text { release }\end{array}$ & $(\mid 40)$ \\
\hline $\begin{array}{l}\text { Rose Bengal } \\
\qquad(\mathrm{RB})\end{array}$ & $\begin{array}{l}\text { Amino-modified MSNs conjugated with RB (MSNs- } \\
\text { RB) }\end{array}$ & In vitro & C. albicans & $\begin{array}{l}\text { Simple RB } \\
\text { conjugation }\end{array}$ & & $(|4|)$ \\
\hline
\end{tabular}


Table III (continued)

\begin{tabular}{|c|c|c|c|c|c|c|}
\hline Drug & Carrier & Assay & Microorganism & $\begin{array}{l}\text { Adsorption } \\
\text { mechanism }\end{array}$ & $\begin{array}{l}\text { Release } \\
\text { mechanism }\end{array}$ & References \\
\hline & & & & & $\begin{array}{c}\text { Slow/con- } \\
\text { trolled } \\
\text { release }\end{array}$ & \\
\hline $\begin{array}{l}\text { Tebuconazole } \\
\text { (TEB) }\end{array}$ & MSNs-N3 (3-[2-(2- & & & & & \\
\hline
\end{tabular}

aminoethylamino)ethylamino]propyltrimethoxysilane)/n vitro

for bacterial infections treatment as well as drugs type, MSNsbased carriers, microorganisms and drugs absorption and release mechanisms.

Bacterial conventional treatment possesses challenges mainly due to the poor antimicrobial compound's permeation into the infected cells. In this regard, Subramaniam et al . (1 10) proposed to evaluate distinct size of MSNs containing rifampicin (RIF) aiming to improve the intracellular bacterial infection treatment. Spherical MSNs were fabricated with average size of 40 and $100 \mathrm{~nm}$ (labeled MSNs-40 and MSNs-100, respectively), zeta potential values of -20 and $-16.9 \mathrm{mV}$, respectively and pores size ranging from 8 to $9 \mathrm{~nm}$. RIF was successfully encapsulated into MSNs-40 and MSNs-100 via passive diffusion method, showing encapsulation efficiency (EE) values of 26.8 and $22.5 \%$, respectively. Cellular uptake assays performed in RAW 264.7 cells exhibited interesting MSNs-100 macrophage internalization achieving up to $80 \%$, whereas MSNs-40 showed lower uptake values (up to $40 \%$ ), assigned to the exocytosis process more pronounced for smaller nanoparticles. From in vitro release assays, both RIFloaded MSNs displayed low release rates up to $10 \%$ (in PBS buffer solution at $\mathrm{pH} 7.4$ ). In contrast, at $\mathrm{pH}$ 5.0, no changes were detected in release profile for both nanosystems within $12 \mathrm{~h}$, attributed to the low RIF solubility. Intracellular infection model was established using small colony variants (SCV) of Staphylococcus aureus in macrophages. Results exhibited high MSNs-RIF internalization and an antibacterial activity enhancement regarding free RIF solution. Authors concluded that despites low release rates achieved, high internalization and bacterial activity enable these nanoplatforms to treat bacterial biofilms.

Regarding bacterial biofilms infections and resistance, Vallet-Regí group $(8,111)$ developed a smart nanocarrier based on antibiotic levofloxacin (LEVO)-loaded MSNs decorated with the lectin concanavalin $\mathrm{A}$ (ConA), as displayed in Fig. 4. ConA was used to aggregate specificity to the nanosystem promoting better internalization into biofilm and then antimicrobial activity. MCM-41-like MSNs with spherical shape of $150 \mathrm{~nm}$ in size and hexagonal pores channels structure were fabricated. In vitro release tests displayed that bare MSNs retained drug release achieving up to $30 \%$ after $48 \mathrm{~h}$. This can be attributed to the hydrogen bonding interaction between MSNs Si-OH groups and LEVO zwitterionic form. On the other hand, $\mathrm{MSN}_{\mathrm{ConA}}$ promoted total LEVO release up to 5 days, assigned to the interaction of ConA protein on MSNs surface with LEVO that leads to its fast release from the mesopores. The nanoantibiotic (labeled $\mathrm{MSN}_{\mathrm{ConA}} @$ LEVO)targeting effect was successfully evaluated in Escherichia coli biofim exhibiting a dose dependent internalization, that means the greater is the $\mathrm{MSN}_{\mathrm{ConA}} @ \mathrm{LEVO}$ concentration, the higher is the nanosystem penetration. The nanoplatform with negatively charged surface (potential zeta of $-25 \mathrm{mV}$ ) promoted high electrostatic affinity toward the biofilm polysaccharide. Antimicrobial in vitro assays were performed in three distinct MSN $\mathrm{ConA}_{1}$ LEVO concentrations (5, 10 and $\left.20 \mu \mathrm{g} \mathrm{mL}^{-1}\right)$. Results confirm high internalization process displaying high antimicrobial efficacy values from 97 to $100 \%$. Beyond that, cytotoxicity assays showed $\mathrm{MSN}_{\mathrm{ConA}} @ \mathrm{LEVO}$ were nontoxic up to $50 \mu \mathrm{g} \mathrm{mL}^{-1}$ enabling these smart nanocarriers for further clinical translations.

Deaconu et al . (112) reported the fabrication of norfloxacin (NFX)-loaded modified and unmodified MCM-type MSNs to evaluate their action against $E$. coli. Authors synthesized five types of MSNs: MCM-41 with long mesopores channels, pristine MSNs without surfactant (MSN), surfactant nonextracted (MSN-E), modified with vinyl groups (MSN-vinyl) and MCM-48 nanosystems. All MCM-type carriers showed spherical nanosized morphologies up to $60 \mathrm{~nm}$. High surface area (up to $1222 \mathrm{~m}^{2} \mathrm{~g}^{-1}$ ) and pores size ranging from 2.5 to $9 \mathrm{~nm}$ were detected for these nanocarriers. MCM-41 types exhibited hexagonal mesopores arrays with average size of 2.5-3.1 nm whereas MCM-48 showed interconnected pores forming cubic arrays with sizes of 2.5-9 nm. The successfully nanoconfinement into mesopores by impregnation method confirmed NFX amorphization. In vitro release studies demonstrated NFX-loaded MSNs carriers enhanced dissolution rates regarding free NFX in same conditions. In addition, the association and dissociation NFX-nanocarriers processes 


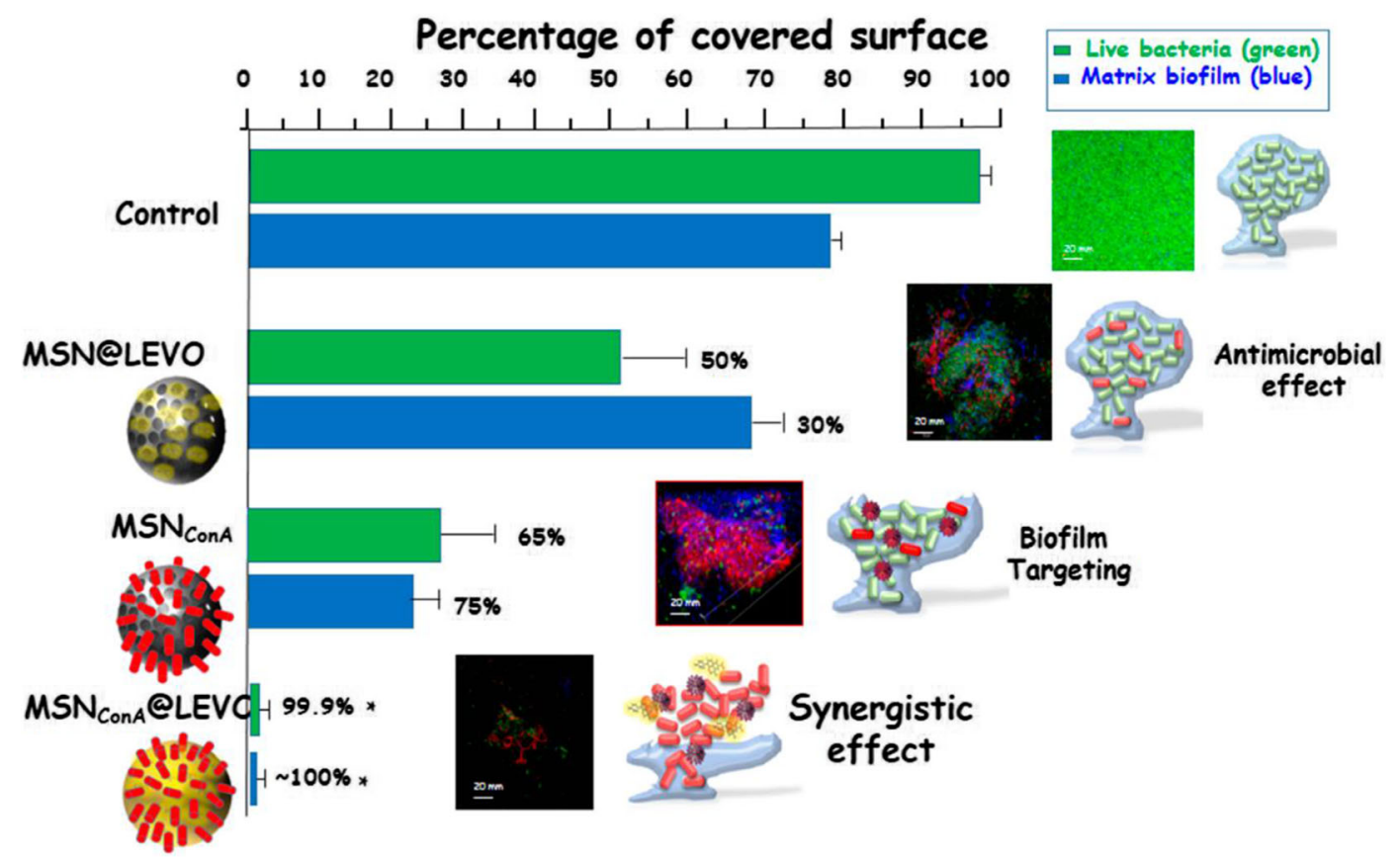

Fig. 4 MSNs as nanocarrier of antimicrobial agent (levofloxacin, LEVO) and functionalized with a biofilm targeting agent (ConA). In this case, the functionalization of MSNs with ConA favours its internalization in E. coli biofilms affording a synergistic combination with LEVO-loaded MSNs, which triggers an efficient antimicrobial effect on E. coli biofilm. The image represents the percentage of covered surface by live bacteria (green) and mucopolysaccharide layer (blue) and the representative confocal images show a complete reduction after incubation with the nanosystems functionalized with ConA and loaded with LEVO (MSNConA@LEVO) [7].

were considered reversible with a first-order kinetics. In addition, high initial release of the antibiotic, which is suitable for applications in severe infections. Authors concluded that NFX-loaded MCM-based nanocarriers displayed good and similar antibacterial activity compared to free NFX. These nanosystems could be applied to avoid the use of high free NFX concentration, maximizing antibacterial effect in any biological media.

Conventional antibiotic treatments are less effective to $S$. aureus because this microorganism can invade host cells like osteoblasts and macrophages and use them as reservoir to reduce antibiotic action leading to infection recurrence. In this sense, Yang et al . (113) proposed to design a gentamicin (GEN)-loaded MSNs modified with a lipid bilayer surface shell and an bacterial-targeting peptide ubiquicidin $\left(\mathrm{UBI}_{29-}\right.$ 41) onto lipid bilayer (labeled LU). Spherical MSNs with highly ordered mesoporous channels and average size of $80 \mathrm{~nm}$ were synthesized. GEN EE of $25.6 \%$ was achieved. MSNs surface modification was firstly performed with liposome layer aiming to prevent nonspecific nanoparticles uptake under physiological conditions whereas the conjugated $\mathrm{UBI}_{29-41}$ allows the nanosystems targeting to bacteria in infected tissues. In addition, the liposomal bilayer can act as a blocker avoiding GEN leakage. When the nanocarrier reaches the infected cells, the liposome can be degraded by bacterial enzymes and toxins. In vitro and in vivo assays were performed on planktonic and intracellular infection of $S$. aureus bacteria. In vitro tests revealed that the $S$. aureus growth on agar plates showed higher microbial inhibition for Gen@MSNs-LU than the unmodified nanosystem (GEN@MSNs) and free drug besides a responsive-bacterial toxin release. From in vivo assays, high specificity, bacterial reduction and no infection recurrence were detected by using GEN@MSNs-LU. In addition, physiopathology studies were performed and no histological changes were detected in major organs indicating no organ toxicity when submitted to GEN@MSNs-LU treatment. All these results enable this bacterial-responsive nanosytem as a promising and adaptable tool to delivery other drugs targeting distinct bacteria and, consequently, treat diverse kind of infections.

Infected bone defects often cause limited blood circulation in the infected sites resulting in poor ability to self-heal for restoring the structure and function of bone tissue. Take this into account, the development of bone implants based on specific and antibiotic controlled delivery systems is essential for an effective bone infection treatment. Zhou et al . (114) designed scaffolds based on MSNs containing vancomycin (VAN) into mesopores channels dispersed in gelatinous matrix. In addition to control drug release, MSNs incorporated into polymer scaffolds can increase their mechanical properties, enhancing cell adhesion, proliferation and osteogenic osteoblasts differentiation. In this context, spherical MSNs were successfully prepared with average size and pores diameter of 210 and $2.4 \mathrm{~nm}$, respectively. In vitro VAN release was 
Fig. 5 Schematic description for the preparation of Ag-Bi@MSNs and its synergistic antibacterial effects [120].

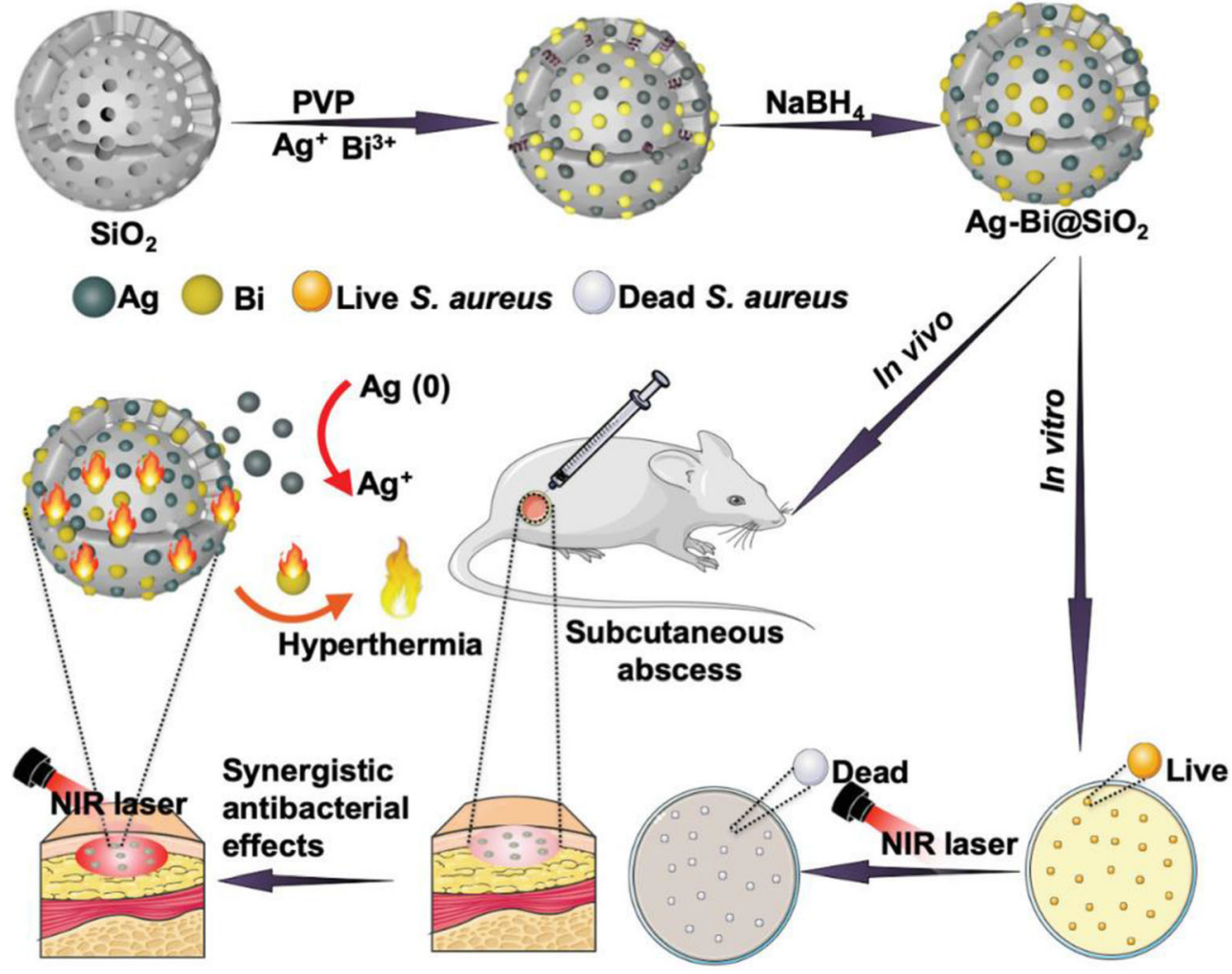

investigated for VAN@gelatin and VAN@MSNs/gelatin. WhileVAN@gelatin displayed an initial burst release (up to 45\% in the first day), VAN@MSNs/gelatin presented an initial release of $19 \%$ (first day) and about 78\% over 28 days, suggesting a sustained VAN release. From in vitro antibacterial ( $S$. aureus) assays, both composite systems showed antibacterial activity dose-dependent with $95 \%$ of growth inhibition at $60 \mu \mathrm{g} \mathrm{mL}^{-1}$. Additionally, cytocompatibility tests were carried out presenting good spreadability and proliferation of bone mesenchymal stem cells (BMSCs) in the evaluated scaffolds. Osteogenic differentiation tests suggested that the incorporation of MSNs facilitated the osteogenic differentiation of BMSGs and VAN-loaded into mesopores had no negative effects on its bioactivity. In vivo tests were established by bone infection induced in rabbits and the implantation of gelatin and MSNs/gelation were evaluated. High leukocytes levels were detected after 8 weeks of treatment for both samples. On the other hand, the implantation of VAN@MSNs/gelatin depicted the leukocyte concentration decreased significantly and reached a normal level after 4 weeks of treatment. The excellent result indicates that MSNs promoted a controlled VAN release at the specific site. Authors concluded that the composite scaffolds based on MSNs as nanocarriers leaded to slow and prolonged VAN release inhibiting local infection in vivo and, consequently, bone regeneration, constituting a promising tool for infected bone defects therapy.

The antibiotic ciprofloxacin (CIP) possesses wide antibacterial spectra and good permeation for bone tissues, however, low blood circulation in bone site, high drug concentration and several side effects makes its direct application inviable. In this context, Andrade et al . (117) fabricated ciprofloxacin (CIP)-loaded SBA-16-type MSNs and SBA-16/hydroxyapatite (HA) composites for bone infection treatment. Both nanoplatforms were modified with 3-aminopropyltriethoxysilane (APTES), anchored with diethylenetriaminepentaacetic acid (DTPA) for chelating technetium-99m. The SBA-16 and SBA-16/HA exhibited ordered cubic mesoporous structures with average pores size of 3.4 and $3.8 \mathrm{~nm}$, respectively. CIP loading ranging from 28 to $31 \%$ were detected for modified and unmodified SBA-16 carriers. In vitro CIP release studies (SBF pH 7.3) displayed that SBA-16-APTES and SBA-16/ HA-APTES were similar with initial burst release of $42-43 \%$ in the first $9 \mathrm{~h}$, achieving the maximum release (51-53\%) in 76 h. These results suggested that at SBF solution with $\mathrm{pH} 7.3$, the nitrogen of piperazinyl ring is protonated and classified as a zwitterionic species, which can lead to great CIP interaction with the amine groups modified onto SBA-16 surface. In vitro antimicrobial assays by using $S$. aureus, Pseudomonas aeruginosa, E. coli and Bacillus cereus were performed for CIP@SBA-16APTES and CIP@SBA-16/HA-APTES. Both aminated platforms presented high antibacterial activity against all microorganisms especially relating to free CIP. It is worth emphasizing that the tested free CIP amount was approximately three times greater than CIP-loaded SBA-16 carriers. ${ }^{99}$ mTc-DTPA-SBA-16-APTES and ${ }^{99}$ mTc-DTPA-SBA-16/ HA-APTES were prepared as radiotracer platforms aiming to 
evaluate their in vivo biodistribution. Both nanosystems were mostly uptaken by liver and spleen, reaching the highest radioactivity level at $4 \mathrm{~h}$ post-injection. Considering bone uptake, a higher uptake for ${ }^{99}$ mTc-DTPA-SBA-16/HA-APTES was detected relating to ${ }^{99} \mathrm{mTc}$-DTPA-SBA-16-APTES, at 1 and $4 \mathrm{~h}$ post-injection. These results suggested ${ }^{99} \mathrm{mTC}$-DTPASBA-16/HA-APTES was more specific for bone tissue than ${ }^{99}$ mTc-DTPA-SBA-16-APTES, which can be attributed to the presence of hydroxyapatite in the nanosystem that possesses high affinity for bone matrix. Authors pointed out that the fabrication of these nanocarriers are relevant showing low cost and easy preparation and effective CIP release, which make them promising nanoplatforms to treat bone infections.

Bacterial biofilm comprises up to $70 \%$ of infection and inflammation in clinic being usually resistant to the most conventional antibiotic treatment. In light of this, $\mathrm{Xu}$ et al . (115) prepared rod shaped hollow MSNs (HMSNs) with large and small cone shaped pores (HMSN-LP and HMSN-SP, respectively) via oil-water system containing lysozyme (LYZ) to treat $E$. coli biofilms. The synthetized nanosystems presented averages particle length and width of 400 and 200 $\mathrm{nm}$, respectively. Average pores size of 40 and $3 \mathrm{~nm}$ were found for HMSN-LP and HMSN-SP, respectively. HMSNLP exhibited loading capacity 7 times higher than HMSN-SP, ascribed to the pores size difference. In vitro release tests (PBS $\mathrm{pH}$ 7.4) revealed that slower drug release was achieved by HMSN-LP than HMSN-SP, with up to $80 \%$ of LYZ release over 48 h. Corroborating the aforementioned results, antimicrobial assays using $E$. coli biofilms model confirmed the highest antimicrobial action of HMSN-LP-LYZ compared to HMSN-SP-LYZ and free LYZ. Additionally, the HMSN-LP was marked with fluorescent dye and its penetration ability into biofilms after 24 and $48 \mathrm{~h}$ of incubation was confirmed by confocal laser scanning microscope (CLSM) analysis. All results demonstrated the high therapeutic activity of HMSNLP towards $E$. coli biofilms, which enable the design of novel and multi-responsive nanosystems against infectious pathogens.

Doxycycline (DOXY) is an antibiotic very hydrophilic and soluble in biological fluid showing a high bioavailability at short time being the most part quickly eliminated by the kidneys. Aiming to overcome the aforementioned drawback and considering the development of bacterial resistance, Deaconu et al . (116) proposed to ally antibiotic properties and MSNs matrix by preparing DOXY-loaded MCM-41type MSNs and modified MSNs to treat Klebsiella pneumoniae strains. MCM-41 (using hexadecyltrimethylammonium bromide $\left(\mathrm{C}_{16} \mathrm{TAB}\right)$ and TMOS as surfactant and inorganic precursor, respectively) platforms were prepared and then modified with phenyl, mercaptopropyl, propylsulfonic and magnesium labeled (MCM-C $\mathrm{H}_{5}$, MCM-SH, MCM-SO $\mathrm{S}_{3} \mathrm{H}$ and MCM-Mg, respectively). MCM- $\mathrm{M}_{14}$ was also synthetized by using reduced chain surfactant $\left(\mathrm{C}_{14} \mathrm{TAB}\right)$ and TEOS as inorganic precursor. All MCM-41 nanoplatforms exhibited spherical shape ranging from 200-400 nm with ordered hexagonal mesopores channels arrays with mean pores size from 1.75 to $2.98 \mathrm{~nm}$. DOXY incorporation was performed for all nanocarriers by wetness impregnation method. From drug release studies (PBS pH 5.2) was detected that DOXY delivery profiles depend on the modification and distinct type of mesoporous silica matrix. The MCM-Mg showed lower DOXY release kinetic, which can be assigned to the enhanced basicity of the magnesium oxide particles formed on the silica surface. Despite distinct DOXY release kinetics obtained, effective and similar antibacterial activity was detected for all nanoplatforms. In addition, these results were similar to free DOXY expected that controlled and prolonged release can be achieved only by using the nanocarriers.

The use of MSNs to transport and deliver poorly soluble drugs to the target diseases sites comprises an attractive and useful method to improve drug's efficacy. Notwithstanding, high loading and also total release drugs amount is a great challenge due to the nanoconfinement and possible interaction with the matrix. In light of this, Chen et al . (118) demonstrated the use of acetophenone (AP), an FDAapproved food additive as co-solvent (known as chaperone) to promotes efficient clofazimine (CFZ) entrapment and release from MSNs channels to aqueous environment. CFZ is a water-insoluble antibiotic used to treat leprosy and multidrugresistant tuberculosis. Spherical and monodisperse MSNs with highly ordered hexagonal mesopores arrays and average size of $100 \mathrm{~nm}$ were obtained as well as high surface area $\left(1060 \mathrm{~m}^{2}\right.$ $\mathrm{g}^{-1}$ ), negatively charged (zeta potential of $-21.2 \mathrm{mV}$ ) with average pore size of $2.8 \mathrm{~nm}$. All these results favor MSNs loading with CFZ and AP. CFZ loading and release assays were carried out in DMSO and AP solvents. The loading CFZ capacity by using AP increased as a function of CFZ concentration, showing $26.8 \%$ at $50 \mathrm{mM}$ whereas by using DMSO the loading capacity was $5.9 \%$ at the same concentration. By using AP strategy, the CFZ release efficiency was $47.2 \%$ whereas by using DMSO was only $0.02 \%$. The novel strategy showed release efficiency 2300 times higher than the conventional method with DMSO $(0.02 \%)$. The high increase in CFZ release can be assigned to the release of AP from the MSNs pores carrying CFZ molecules together into the aqueous solution. The chaperon-based MSNs were evaluated in a macrophage model of Mycobacterium tuberculosis infection. The optimized CFZ-AP(10mM CFZ in AP)@MSNs exhibited high antimicrobial efficacy (dose-dependent) reducing the bacteria number by $1 \log$ CFU (90\% of bacteria were killed). In addition, no changes on the macrophages morphological appearance were detected for the applied nanosystems. This loading/delivery "chaperone-assisted" strategy can be used for other hydrophobic drugs with their suitable solvents (chaperone), opening up opportunities to design novel drug delivery MSNs-based nanosystems for biomedical applications. 
Despites the challenges cause by microbial biofilms formation and antibiotic resistance generation, Gram-negative bacteria possess outer membranes as a highly impermeable barrier as well as additional defense mechanisms no detect in Gram-positive bacteria (153). Considering these drawbacks, González et al . (119) developed nanoantibiotics based on levofloxacin (LEVO)-loaded MSNs covalently modified with third-generation poly(propyleneimine) dendrimer $(\mathrm{G} 3)$, which has high affinity to negatively charged bacteria cell walls. Beyond that, LEVO-loaded [3-(2-aminoethylamino) propyl]trimethoxysilane (labeled DAMO)-modified MSNs (noted LEVO@MSNs-DAMO) were also fabricated to evaluate antimicrobial action against Gram-negative bacteria. Sphericalshaped MSNs with 2D hexagonal mesoporous arrangement were prepared, showing average diameter and pores size of 150 and $2.4 \mathrm{~nm}$, respectively. In addition, negatively charged bare MSNs (-36.4 $\mathrm{mV}$ ) after modification become positively charged achieving zeta potential around $+31.8-37.4 \mathrm{mV}$, which make them enable to interact with negatively charged bacteria walls. LEVO loading amount up to $7.8 \%$ were detected for the nanovehicles. LEVO release performance (PBS pH 7.4) were evaluated exhibiting a first-order kinetics for all nanosystems (LEVO@MSNs, LEVO@MSNs-DAMO and LEVO@MSNs-G3), with 100\% of LEVO release over 72 h for LEVO@MSNs-G3. LEVO@MSNs showed lower release values indicating strong interaction via hydrogen bonding between LEVO zwitterionic form (at pH 7.4) and Si-OH groups from MSNs. E. coli internalization assays confirmed good MSNs-DAMO and MSNs-G3 internalization due to their positive charge density making electrostatic interactions with $E$. coli cells walls possible. Additionally, MSNs-G3 displayed higher internalization than MSNs-DAMO, ascribed to the MSNs-G3 high surface flexibility and high interaction points with negatively charged $E$. coli phospholipid membranes regarding MSNs-DAMO. These results corroborated antimicrobial assays against $E$. coli biofilms showing the MSNs-G3 with higher and remarkable antimicrobial efficacy relating to the other nanosystems. These findings demonstrated that the synergistic combination of bacterial internalization and antimicrobial agents into MSNs-based nanovehicles lead to interesting and efficient antibiotics for infections treatment.

Regarding the high mortality and morbility caused by bacterial resistance, Cao et al . (120) proposed to ally the amazing properties of MSNs with metallic nanoparticles such as silver $(\mathrm{Ag})$ and bismuth (Bi). Authors designed a multifunctional MSNs-suported Ag-Bi nanoparticles (NPs) associating hyperthermia generated by Bi nanoparticles with $\mathrm{Ag}$ release for synergistic antibacterial therapy as shown in Fig. 5. Ag-Bi nanoparticles were fabricated via in situ growth onto MSNs pores structure aiming to avoid their aggregation and oxidation under physiological environment. Under the NIR laser irradiation (808 $\mathrm{nm}$ laser irradiation, $1 \mathrm{~W} \mathrm{~cm}^{-2}, 15 \mathrm{~min}$ ), hyperthermia can be generated by Bi NPs destroying bacterial cell membrane and biofilm promoting and increasing $\mathrm{Ag}^{+}$ release from the nanocarrier, resulting in photothermalenhanced antibacterial activity. Spherical nanosystems with average size and pores of 200-240 nm and 3.4-4.8 nm, respectively were successfully fabricated. In addition, Ag NPs showed average size of $\sim 15 \mathrm{~nm}$. Photothermal therapy (PTT) ability of Ag-Bi@MSNs was evaluated exhibiting high stability and laser power irradiation and concentration dependence. As higher is laser power density and Ag-Bi@MSNs concentration, higher is the achieved temperature confirming great PTT performance. In vitro antibacterial activity was evaluated against MRSA cells by adding Ag-Bi@MSNs with or without laser irradiation. At same concentration $(128 \mu \mathrm{g} \mathrm{mL}$ $\left.{ }^{1}\right), 22$ and $76 \%$ of cells death were achieved without and with laser irradiation, respectively. Additionally, release studies were performed for $75 \mathrm{~min}$ with and without laser irradiation (at the same concentration, $200 \mu \mathrm{g} \mathrm{mL}^{-1}$ ). Results corroborate antibacterial assays displaying a NIR-responsive $\mathrm{Ag}^{+}$release, with 11.3 and $37.3 \%$ of release without and with laser irradiation, respectively. Aiming to prove the synergic antibacterial effect, MRSA-infected mice were investigated with five groups: PBS, Bi@MSNs, Ag-Bi@MSNs, Bi@MSNs + NIR and Ag-Bi@MSNs + NIR groups. Regarding to the control group (PBS), the number of colonies were reduced to 90.3, 74.6, 35.1 and 5.9\% for Bi@MSNs, Bi@MSNs + NIR, AgBi@MSNs and Ag-Bi@MSNs + NIR, respectively, indicating that multimodal synergistic therapy based on Ag-Bi@MSNs could effectively treat MRSA skin infection. The therapeutic effect was confirmed by histological analysis. Beyond that, the animals evaluated showed no obvious changes in their body weight suggesting no biotoxicity. These findings made AgBi@MSNs a promising multimodal nanoplatform against bacterial infections and for further clinical translations.

Fast and accurate bacteria detection and their effectively killing in the bloodstream in low doses are crucial and act as key roles in medicine and microbiology studies. Integrating diagnosis and treatment to achieve accurate diagnosis and efficient treatment of diseases has always been an important goal of modern medicine. In this context, $\mathrm{Xu}$ et al . (122) developed a smart and theranostic nanoplatform based on vancomycin (VAN)-loaded magnetic $\left(\mathrm{Fe}_{3} \mathrm{O}_{4} \mathrm{NPs}\right) \mathrm{MSNs}$ (MMSNs) modified with sufonated-hyaluronic acid (S-HA) and $S$. aureus antibody $(\mathrm{Ab})$ grafting via amidation reaction (labeled Ab@S-HA@MMSNs) for S. aureus detection and treatment in bloodstreams. S-HA was selected due to its capping and targeting function, biocompatibility, biodegradability besides promotes drug sustained release and anti-adhesion effects. $\mathrm{Fe}_{3} \mathrm{O}_{4}$ NPs displayed average size of $180 \mathrm{~nm}$ whereas spherical MMSNs achieved average size and pores diameter of 240 and 3 nm, respectively. In addition, Ab@SHA@MMSNs exhibited 180 nm core uniformly wrapped with $30 \mathrm{~nm}$ shell, with zeta potential of $-5.32 \mathrm{mV}$. Van loading efficacy of $10.7 \mathrm{wt} \%$ was achieved and antibacterial assays 
were carried out since $S$. aureus can secrete hyaluronidase (Hyal), enzyme that degrades S-HA promoting controlled VAN release. Results exhibited $98 \%$ of bacterial reduction suggesting high antibacterial efficacy and Hyal-responsive controlled VAN release. In vitro release tests were conducted showing that no significant VAN was release without Hyal presence whereas after Hyal addition (up to $500 \mathrm{U} \mathrm{mL}^{-1}$ ), up to $83 \%$ of VAN corroborating the Hyal role in the "ondemand"drug release. The Ab@S-HA@MMSNs magnetization behavior was evaluated and despites magnetization values reduction after all modification, the nanosystem presented good magnetic responsivity and can be utilized to modify the surface of magnetic glassy carbon electrode (MGCE) (noted Ab@S-HA@MMSNs/MGCE) by magnetic interaction. From electrochemical and analytical studies, the fabricated immunosensor exhibited an excellent electrochemical response towards $S$. aureus in the range of $1 \times 10^{1}-1 \times 10^{10}$ CFU $\mathrm{mL}^{-1}$ with high selectivity, stability and reproducibility. The detection limit with linear range was up to $3 \mathrm{CFU} \mathrm{mL} \mathrm{m}^{-1}$, and this responsiveness was also well demonstrated in the blood environment. Beyond that S-HA-modified MMSNs possess anticoagulant property, the Ab@S-HA@MMSNs/ MGCE displayed antiadhesion property, ensuring that it can be directly applied to detect $S$. aureus in whole blood. Additionally, increasing $S$. aureus amount of arriving at Ab@S-HA@MMSNs/MGCE, the cappingAb@SHA@MMSNs were degraded by Hyal secreted by $S$. aureus and "on demand" VAN released was achieved. All results suggest this multifunctional theranostic nanoplatform for accurate diagnosis and efficient treatment of $S$. aureus bloodstream infection.

Zink group $(23,118,125,132-135)$ have designed stimuliresponsive controlled drug delivery $\mathrm{MSN}_{s}$-based nanoplatforms to treat infectious diseases such as caused by Francisella tularensis $(125,130,134,135)$ and $M$. tuberculosis $(118,132,133)$. From one of these works (135), authors developed a pathogenspecific detection and drug delivery nanoplatform based on MSNs modified with silane groups attached with lipopolisaccharide chain of $F$. tularensis live vaccine strain (Ft-LVS-LPS) as stalks (modified antigen) that interact with FB11 anti-Oantigen antibody (FB11) nanovalve as a capping agent to block the mesopores avoiding drug diffusion without specific stimulus. By manipulation of the non-covalently interaction MSNs modified antigen (Ft-LVS-LPS)-antibody (FB11), the antigen produced by the target bacteria can compete effectively for binding to the gatekeeper antibody, reducing stalknanovalve interaction, leading to displacement of the antibody, pore uncapping and finally cargo release. Model drugs such as fluorescein or Hoechst 33342 were used to evaluate the nanoplatform specificity and action. Spherical MSNs with high surface area $\left(840 \mathrm{~m}^{2} \mathrm{~g}^{-1}\right)$, surface charge of $+36 \mathrm{mV}$ and average diameter and pores size of 117 and $3.8 \mathrm{~nm}$, respectively, were prepared as nanoplatform. The specific antigen- antibody modification was successfully confirmed by immunostaining assay showing no nonspecific binding of the FB11 antibody to the unfunctionalized MSN surface and also no nonspecific binding of the fluorescent secondary goatantimouse (GAM) immunostaining antibody to the unfunctionalized MSN surface or the Ft-LVS-LPS. Authors also evaluated whether a competitive FB11 antibodies displacement could be achieved in the presence of Ft-LVS-LPS. From immunostaining assays, by using both types of silane groups (APTES and ICPTES) as stalks to interact with the modified antigen, successful displacement of FB11 antibodies from the MSN surface in the presence of Ft-LVS bacteria can be confirmed in vitro indicating that the operation of the nanovalve is feasible at high bacterial concentration. In addition, the reduction of the FB11 antibody affinity to the LPSmodified MSNs surface was achieved by acetylation of LPSmodified MSNs reducing the $\mathrm{OH}$ groups available for hydrogen-bonding with FB11, requiring lower Ft bacterial concentrations to displace FB1 1 antibody from the nanoplatform. From cargo release studies, authors reported that the use of LPS from a Ft wzy deletion mutant can promote better mesopores capping avoiding any cargo leakage before reach the target site. Beyond that, from this highly specific nature of the antibody-antigen interaction, a significant cargo release was detected only in Ft-LVS-LPS presence. These results suggested that the pathogen-sensitive controlled cargo release nanoplatforms can be also applied to other gram-negative bacteria as novel theranostic smart MSNs-based nanosystem for infectious diseases treatment.

\section{Parasitic Infections}

MSNs have received great attention towards vaccines production due to the large amount of silanol groups on its surface that facilitates the functionalization and drugs incorporation. The surface functionalization with several molecules allows improving cells target and uptake besides favor drugs transport and release from the mesopores, leading to interesting immune and cellular responses, which makes MSNs attractive and potential nanoplatforms for vaccines fabrication against any infectious diseases (154).

Few studies have been reported in the literature applying MSNs for parasitic diseases treatment (as shown in Table III). For instance, Oliveira et al . (136) described that vaccine and prophylactic protection development against Schistosoma mansoni are essential to reduce the infection cases. In this way, authors reported the use of MSNs containing SWAP (Antigenic Preparation of Soluble Worm) as new strategy to treat $S$. mansoni infection. MSNs were characterized according to their size $(38.8 \mathrm{~nm})$, polydispersity index $(0.24)$, zeta potential $(-32.9 \mathrm{mV})$, surface area $\left(871.19 \mathrm{~m}^{2} \mathrm{~g}^{-1}\right)$ and porosity with pores volume and diameter of $0.243 \mathrm{~cm}^{3} \mathrm{~g}^{-1}$ and $6.91 \mathrm{~nm}$, respectively. SWAP was successfully incorporated into MSNs 
showing EE up to $35.35 \%$. Immunological tests indicated that MSNs were able to stimulate a high immune response compared to conventional adjuvants (SWAP-associated aluminum salt). Authors also reported that higher immunization performance observed for MSNs may be related to some unique characteristics presented by the nanosystem, such as high surface area, spherical morphology, high pores volume, stability, biocompatibility and non-toxicity which make SWAP-loaded MSNs a promising strategy to improve immune response against $S$. mansoni and enabling vaccines production.

In another study, Nhavene et al . (137) designed multifunctional MSNs based on MCM-41 functionalized with (3glycidoxypropy) trimethoxysilane (GPTMS) and chitosan succinate (CS) containing benznidazole (BZ) onto modified MSNs surface aiming to evaluate their action against the parasite Trypanosoma cruzi responsible for Chagas disease. The MSNs-like MCM-41 were successfully prepared with welldefined hexagonal arrays of uniform mesopores network, spherical shape and pores size of $3.3 \mathrm{~nm}$. The MSNs functionalization with GPTMS and CS was confirmed by spectroscopic analysis. BZ anchored onto CS-modified MSNs surface via hydrogen bond interactions was confirmed by spectroscopic and density functional theory (DFT) analysis. In vitro assays showed that the nanosystem can effectively performed BZ delivery being thirty times more active against epimastiogotes T. cruzi CL-Brener than free BZ, revealing its potential and effectiveness as nanocarrier for BZ delivery. Authors also concluded that all results demonstrated MSNs-GPTMS-CS as a potential and promising nanoplatform for drugs and genes delivery to treat neglected diseases such as Chagas disease or American trypanosomiasis.

Tawfeek et al . (138) fabricated praziquantel (PZQ)-loaded MSNs in order to evaluate its therapeutic effect against murine S.mansoni.MSNs@PZQshowed spherical shape, average size ranging from 100 to $105 \mathrm{~nm}$, zeta potential from +31.9 to $+30 \mathrm{mV}$ and $\mathrm{EE}$ of $83 \%$. Mice were infected with S. mansoni and treated 6 weeks after infection with similar doses of MSNs@PQZ and free PZQ evaluating several concentrations, orally and intraperitoneally (IP). A maximum antischistosomal effect was achieved using MSNs@PZQ administered orally. The biomarkers related to liver oxidative stress status and immunomodulatory effect (serum TNF- $\alpha$ and IL10) were significantly improved showing IP route was less effective for PQZ delivery. Authors also reported that the MSNs@PQZ administration allows the reduction of the effective therapeutic dose, indicating MSNs as a safe nanoplatform that enhances anti-schistosomal, antioxidant, immunomodulatory and anti-inflammatory PQZ actions in animal model infected by $S$. mansoni.

Yunessnia lehi et al . (139) synthesized whisker-formed SBA-15 nanoparticles as a pH-sensitive metronidazole (MNZ)-trapped nanocarrier. This nanosystem was modified with tannic acid (TA) aiming to block the mesopores and control drug release process. The SBA-15 nanowhiskers had small fibrous structures $0.5-1.5 \mu \mathrm{m}$ in length and up to $50 \mathrm{~nm}$ in diameter. Through characterization analysis, 2D ordered hexagonal mesostructures were detected, with surface area of $491.38 \mathrm{~m}^{2}$ and pores volume and diameter of $0.593 \mathrm{~cm}^{3} \mathrm{~g}^{-1}$ and $6.06 \mathrm{~nm}$, respectively. In addition, the modification with TA increased MNZ EE from 62.6 to $71.4 \%$. In vitro release assays exhibited fast MNZ release from unmodified SBA-15 nanowhiskers whereas TA-SBA-15 nanocarriers controlled MNZ release for an extended time. Interestingly, the modified nanosystems displayed a $\mathrm{pH}$-sensitive behavior, showing MNZ release up to $85 \%$ at $\mathrm{pH} 5.8$. The minimal lethal concentration $\left(0.5 \mathrm{mg} \mathrm{mL}^{-1}\right)$ of TA-modified SBA-15@MNZ showed $100 \%$ protozoal growth inhibition for $180 \mathrm{~min}$ regarding to parasite control and free MNZ solution. These studies suggest this multifunctional nanoplatform as promising and efficient MNZ delivery system for parasitic diseases treatment.

\section{Fungal Infections}

The development of new formulations against Candida albicans is essential to overcome drawbacks as multiple resistance mechanisms developed by biofilms formation, which makes this pathogen resistant to drugs such as fluconazole and amphotericin B, and, consequently, making difficult to treat effectively these infections, contributing to high morbidity and mortality rates in immunocompromised patients $(141,155)$. In light of this, Paramanantham et al . (141) reported a nanosystem based on MSNs modified with Rose Bengal (RB) as photodynamic therapy (PDT) as antimicrobial alternative to treat C. albicans biofilms. MSNs with average pores size and diameter of 2 and $500 \mathrm{~nm}$, respectively, were amino functionalized aiming to increase the conjugation capacity of RB. RB is a photosensitizer, an organic compound that absorbs light of a suitable wavelength generating singlet oxygen and reactive oxygen species (ROS), which can kill microbial systems. High values of RB EE (58.54\%) was achieved by amino-modified MSNs due to the good electrostatic interaction of the positively charged MSNs surface with anionic dye RB. Taking the aforementioned into account, in vitro release tests showed a slow and controlled RB release with a maximum of $66.38 \%$ after 3 h. Additionally, cellular uptake studies exhibited high MSNs-RB uptake (up to $47 \%$ ) compared to the free RB (up to $10 \%$ ) after $3 \mathrm{~h}$. Through in vitro release results, amine groups modified onto MSNs enabled a slow and controlled release, by electrostatic attraction with the RB, which is desirable and allow a good PDT action after a single administration. When associated with PDT, high values of MSNs-RB antimicrobial action were achieved for both $C$. albicans planktonic cells (up to $88.7 \%$ ) and biofilms (up to $79.7 \%$ ) regarding pristine MSNs and free RB. Beyond that, after light irradiation, MSNs-RB exhibited high ROS production, proteins leakage (from 
cytoplasm C. albicans cells), DNA damage and lipid peroxidation. These results confirmed the MSNs-RB as an efficient nanosystem as antimicrobial PDT against infections caused by antifungal drug resistant and biofilm forming strains, being its application extended to enhance $C$. albicans eradication on medical devices such as implants and catheters.

Econazole (ECO), an antifungal widely used for topical infections treatment has limited bioavailability due to its low aqueous solubility (156). Aiming to overcome ECO solubility drawbacks, Montazeri et al . (140) developed ECO-loaded MSNs modified with aminopropyl groups. Spherical shape and hexagonal mesopores arrays were detected for MSNs. Pristine MSNs, modified MSNs and ECO-loaded MSNs displayed average size ranging from 39 to $91 \mathrm{~nm}$ while the nanosystems presented average pores size up to $2.54 \mathrm{~nm}$. In addition, MSNs was negatively charged (MSNs and MSNs-ECO showed -12.14 and $-17.51 \mathrm{mV}$, respectively) whereas after amino modification the nanosystem showed positive surface charge $\left(\mathrm{MSN}_{s}-\mathrm{NH}_{2}\right.$ and $\mathrm{MSN}-\mathrm{ECO}-\mathrm{NH}_{2}$ exhibited +32.52 and $+33.63 \mathrm{mV}$, respectively). $\mathrm{MSNs}$ and $\mathrm{MSNs}-\mathrm{NH}_{2}$ exhibited ECO EE of up to 42 and $63 \%$ and release rate of 29.57 and 38.65, respectively. Both MSNs and modified MSNs containing ECO were non-toxic to human dermal fibroblast strains cells. Distinct ECO/MSNs and modified MSNs ratios were evaluated being that the antifungal activity (C. albicans) for all nanosystems increased as drug amount loading increased. Greater antifungal activity was observed for modified nanosystems compared to the non-modified ones showing inhibition zone of up to 19.5 and $16.0 \mathrm{~mm}$, respectively (at $1.2 \mathrm{mg} \mathrm{mL}^{-1}$ of ECO). ECO-loaded MSNs displayed greater antifungal activity regarding to the econazole cream, confirming the nanosystem efficiency for the infectious treatment caused by fungus such as $C$. albicans. Additionally, the developed nanosystem exhibited non induced skin irritation on rabbits, being cutaneous reaction negligible after $72 \mathrm{~h}$. It is worth mentioning that the designed nanoplatform could reduce the treatment duration by increasing patient compliance, making this nanosystem desirable for fungal therapy purposes.

In another study, authors (142) fabricated pH-responsive gated MSNs-based nanosystem aiming to treat fungal infections such as vaginal candidiasis. The sensitive MSNs like MCM-41 nanoplatforms were loaded with tebuconazole (TEB) and modified with silane groups as 3-[2-(2-aminoethylamino)ethylamino]propyltrimethoxysilane (N3) used as gatekeepers that can avoid TEB leakage from the mesopores before the nanosystem reach the target site. Spherical-shaped silica nanoplatform with average size of $100 \mathrm{~nm}$ and hexagonal pores arrays with mean diameter of $2.45 \mathrm{~nm}$ were successfully prepared as well as the TEB loading $\left(0.064 \mathrm{mmol} \mathrm{g}^{-1}\right.$ of MSNs) into mesopores and subsequently capping with N3 groups (2.43 $\mathrm{mmol} \mathrm{g}^{-1}$ of MSNs). For cellular uptake assays, fluorescein (FL, green dye) were loaded into MSNs and modified with the N3 gatekeeper. The simulated nanosystem exhibited good cellular internalization (Saccharomyces cerevisiae cells, incubated at pH 3.7) detected by fluorescence microscopy. In addition, in vitro release FL tests were performed at $\mathrm{pHs}$ 3.7 and 5.5. Results showed that more than 95\% of FL was released after $12 \mathrm{~h}$ whereas almost no release was detected at $\mathrm{pH} 3.7$, confirming the design of $\mathrm{pH}$-responsive nanosystem. At $\mathrm{pH} 3.7$, the polyamines of $\mathrm{N} 3$ capping was more protonated than at $\mathrm{pH} 5.5$, which allowed high rigid chain conformation and high interactions with anions $\left(\mathrm{as}_{4}{ }^{2-}\right)$, that favor the mesopores blockage. At pH 5.5, the polyamine chains is more flexible allowing pores opening and FL release. After successful cellular uptake and FL release tests, in vitro antifungal assays (C. albicans cells) were performed with the MSNsTEB-N3 and free TEB. Results exhibited high antifungal activity promoting $90 \%$ of cells death by using MSNs-TEB-N3 while free $\mathrm{TB}$ showed only $10 \%$ of action. In this way, the designed smart nanoplatform improved TEB efficacy comprising an interesting tool to overcome side effects associated with topical therapies for vulvovaginal infection and improve its cost-effectiveness. A schematic $\mathrm{pH}$-responsive nanosystem action is displayed in Fig. 6.

In order to facilitate metallic nanoparticles (MNPs) applications as multifunctional drug delivery systems, authors $(143,144,157)$ have proposed their association with MSNs aiming to increase biocompatibility avoiding MNPs aggregation and action lost. Mitra et al . (143) incorporated zinc oxide nanoparticles (ZNPs) into MSNs (noted $\mathrm{ZnO} @ \mathrm{MSNs}$ ) in order to evaluate the antifungal efficiency against two strains of Aspergillus niger (MTCG 10180 and MTCG 2196) and two strains of Fusarium oxysporum (NCIM 1043 and NCIM 1072). The MSNs and ZnO@MSNs nanosystems displayed spherical shape with a size ranging from 20 to $40 \mathrm{~nm}$ with average pore size ranging from 2.5 to $3.1 \mathrm{~nm}$, respectively. The authors observed that $\mathrm{ZnO} @ \mathrm{MSN}$ activity was dose dependent, requiring high doses to achieve antifungal response. The enzymatic tests showed that $\mathrm{ZnO} @ \mathrm{MSN}_{\mathrm{S}}$ activity occurred through the generation of oxidative stress through ROS generation. The generated oxidative stress caused morphological changes in the fungi. Both in vitro and in vivo tests indicated good biocompatibility of the developed nanosystem, with sustained release exerted by MSNs preventing the $\mathrm{ZnO}$ direct exposure reducing toxic effects, which makes the developed MSNs-based nanoplatform enable to desirable antimicrobial applications.

In another study, silver nanoparticles $(\mathrm{AgNPs})$, synthesized by green synthesis using Azadirachta indica leaf extract as a reducing agent, were loaded into MSNs (noted MSNsAgNPs) aiming to evaluate antifungal activity against C. albicans 077 strains. The fabricated MSNs presented spherical shape with size of around $400 \mathrm{~nm}$ and hexagonal pores structures with average size of $\sim 7.6 \mathrm{~nm}$. The impregnated AgNPs were detected onto MSNs pores/surface showing spherical shape with average size ranging from 5 to $50 \mathrm{~nm}$. 
Fig. 6 Schematic representation of the design and action mechanism of $\mathrm{SI}$-Teb under usual vaginal conditions $(A)$ and in the presence of C. albicans (B) (S. cerevisiae has been used in experimental assays as a model organism). Abbreviation: MCM-4I, mobil composition of matter-4I; SI-Teb, MCM-4I loaded with tebuconazole [142].
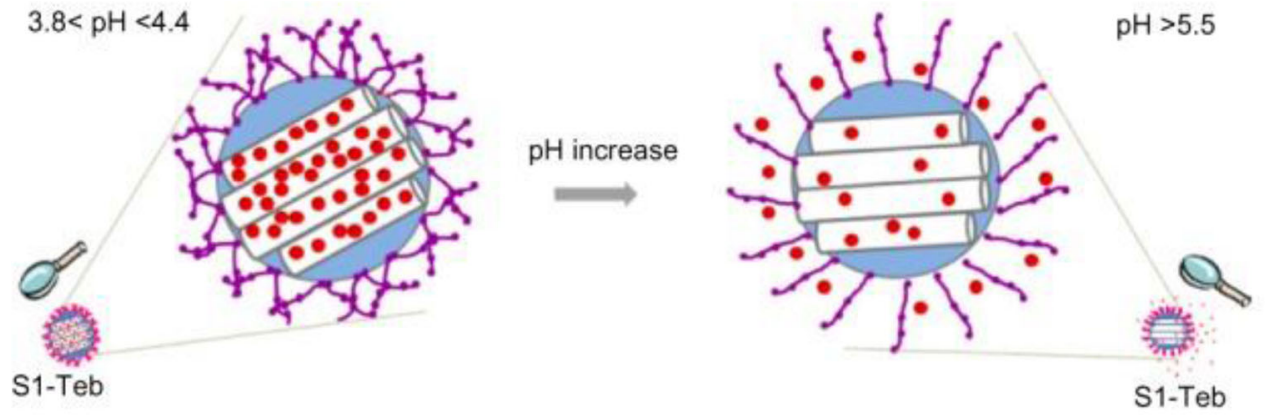

a

b

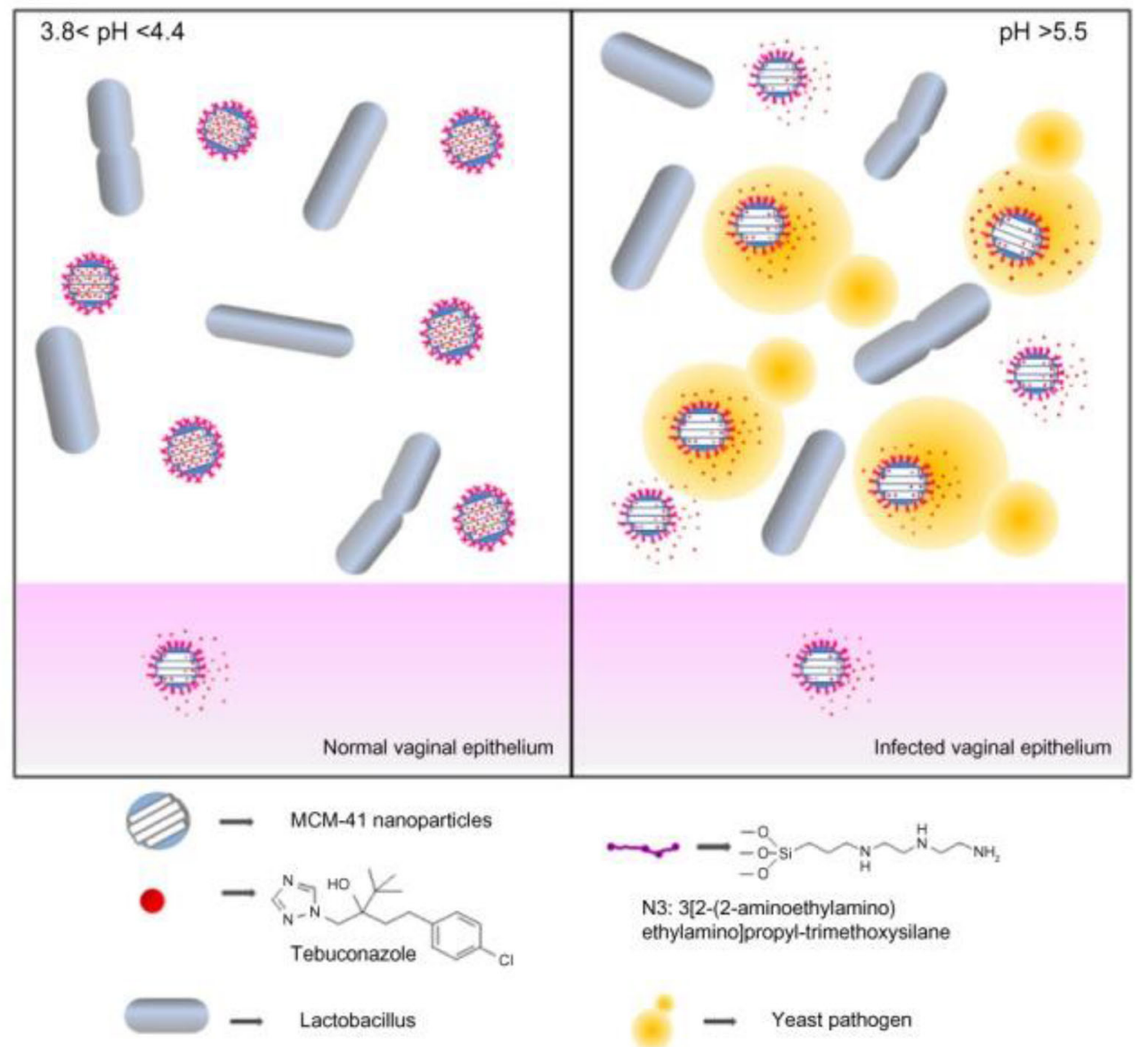

From antifungal activity assays, MSNs-AgNPs proved to be efficient in inhibiting the fungus growth in a dose-dependent manner, both in the disc diffusion and in vitro killing assays, being a promising alternative approach to infections treatment caused by $C$. albicans. However, more preclinical studies are already essential to better understand the real potential of the MSNs-AgNPs for future clinical translations (144).

\section{Viral Infections}

Viral infections and spreadability can be establish only by their interaction with host cells. The key and great challenge to develop viral infections treatment consist on understand and elucidate the interaction virus-host cells at molecular level. In addition, virus can evolve by genetic mutation and become drug resistant making urgeous and essential the design of new drugs. In current medical scenario, the lack of antiviral broad-spectrum drugs, the fast and wide spreadability and the long time demanded to elucidate virus mechanism action to design a safety and efficient vaccines or treatment making viral infections such as SARS-CoV-2 a great threat to human global health $(6,146,147,158,159)$.

Taking the aforementioned into account, the design of new antiviral MSNs can be an effective approach to control viral 
infections inhibiting virus mechanism action by interaction with virus, targeted drug delivery, interaction with host cells receptors inducing cellular immune response $(6,146,147,158,159)$.

Venezuelan equine encephalitis virus (VEEV) comprises a great public health concern due to its possibility amenability for use as a bioterrorism agent and its severe health consequences in humans. In addition, natural VEEV outbreaks resulted in equine and human infections, provoking high death rates in equines (85\%) and chronic neurological complications in humans. A chemical VEEV inhibitor known as ML336 was developed and its effectiveness in VEEV infection was confirmed in preclinical assays. However, the great limitation for ML336 clinical translation consists on its poor solubility and stability in biological media. Taking this into account, LaBauve et al . (145) designed lipid-coated MSNs (LC-MSNs) as smart nanocarriers for ML336 delivery, as depicted in Fig. 7. This nanovehicle was proposed to improve ML336 stability, promoting its controlled release for VEEV treatment. Narrow size distribution $(\sim 75 \mathrm{~nm})$ and hexagonal pores structures (average pore size of $2.65 \mathrm{~nm}$ ) were achieved for MSNs. The lipidic shell consists of a liposome structure composed by 1,2-distearoyl-sn-glycero-3-phosphocholine (DSPG):Cholestrol:1,2-distearoyl-sn-glycero-3-phosphoethanolamine-N-[methoxy(polyethylene glycol)-2000] (DSPEPEG(2000)), which can enhance colloidal stability, circulation time besides reduce protein adsorption to the nanosystem surface. From electron microscopy analysis, LC-MSNs@ML336 showed a lipid bilayer thickness of $6 \mathrm{~nm}$ besides zeta potential of $-1.76 \mathrm{mV} .20 \mu \mathrm{g}$ ML336/mg LC-MSNs were loaded into the nanocarrier showing a ML336 release of 34\% over $24 \mathrm{~h}$. TC-83 VEEV infected HeLa cells were treated with LCMSNs@ML336. Results displayed virus inhibition in a dosedependent manner, with significantly decreased viral load by 4 orders of magnitude after $24 \mathrm{~h}$ and 6 orders of magnitude after 48 and 72 h. From in viwo assays, LC-MSNs were considered nontoxic at $0.11 \mathrm{~g}$ LC-MSNs/ $\mathrm{kg}$ mouse dose administered per day during four days. In addition, LCMSNs@ML336 exhibited significant reduction (about 10fold) of brain viral titer in VEEV infected mice compared to PBS and other controls. These results highlighted the LCMSNs@ML336 potential to treat VEEV infections and LCMSNs as smart nanovehicles for antiviral applications.

Herpes simplex virus (HSV) provokes several infectious diseases such as orolabial and genital herpes being currently treated by guanine analogues such as acyclovir, valaciclovir and others, which inhibit viral DNA replication. However, these compounds suffer from antiviral drug resistance especially in immunocompromised individuals besides show poor bioavailability being necessary several and high doses administration (146,147). The HSV cells infection can start by electrostatic and hydrophobic interactions between viral glycoprotein (gB or gC) and cell glycosaminoglycans (GAGs, heparan sulfate) composed of negatively charged sulfonate groups linked by hydrophobic moieties. Molecules that mimic GAGs can interact with virus avoiding their interaction and penetration into susceptible cells $(146,147)$. In this context, Le et al . $(146,147)$ have designed GAG mimetic-functionalized MSNs to treat HSV-1 and HSV-2 infections. In a first work (146), aryl sulfonate GAG mimetic-modified MSNs exhibited low
Fig. 7 Schematic representation of LC-MSNs@ML336. The antiviral ML336 was incubated with MSNs prior to vesicle fusion with liposomes containing a composition of $77.5 \%$ DSPC: $2.5 \%$ DSPEPEG2000:20\% cholesterol at mole ratios [I 45].

\section{LIPID VESICLE COMPOSITION}

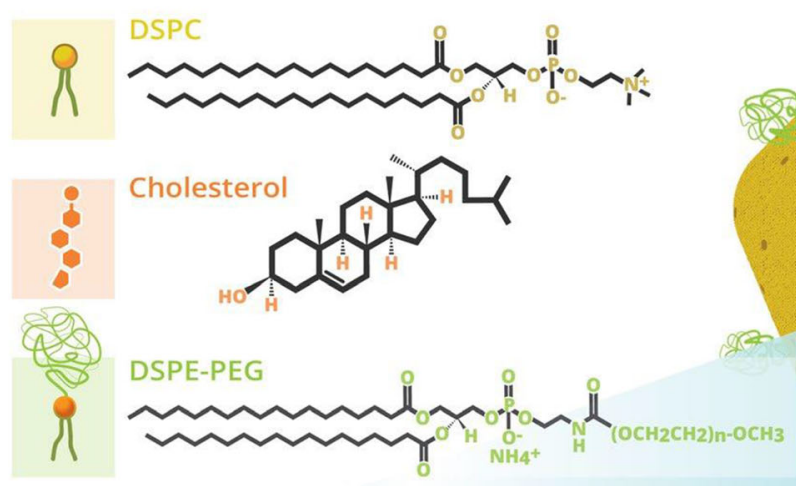

SUPPORTED LIPID BILAYER

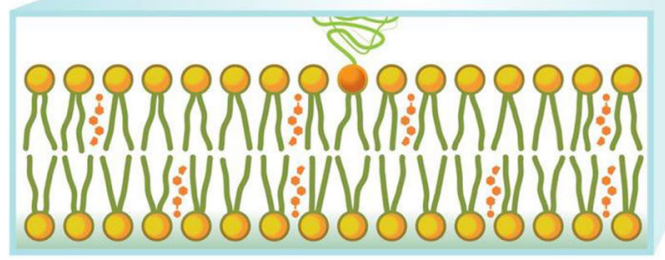

LC-MSN
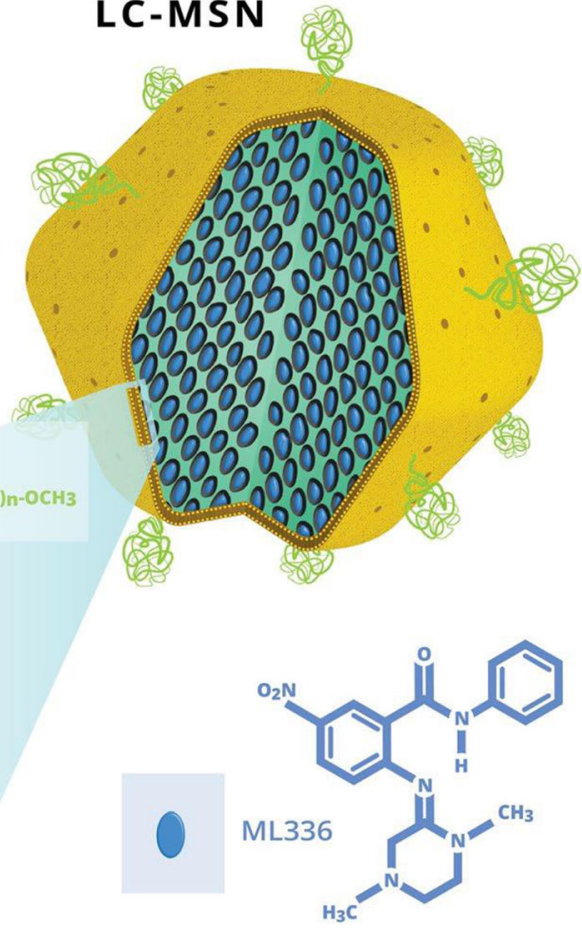
toxicity besides inhibited $\mathrm{HSV}-1$ and $\mathrm{HSV}-2$ penetration into health cells while controls were inactive. In this work, Lee et al . (147) explored distinct functional groups related to GAG structure attached to MSNs and investigated their activity against HSV-1 and HSV-2. In addition, authors evaluated GAG mimetic-functionalized MSNs as smart nanocarriers for acyclovir (ACV) delivery aiming simultaneously inhibition of viral penetration and DNA replication. Five modified MSNs were prepared: benzene sulfonate-modified MSNs, propyl thiol-modified MSNs, propyl sulfonate-modified MSNs, zwitterionic sulfonate-modified MSNs and phenylmodified MSNs ((MSNs-Phenyl-SO ${ }_{3}$, MSNs-Propyl-Thiol, MSNs-Propyl-SO ${ }_{3}, \mathrm{MSN}_{\mathrm{N}} \mathrm{ZW}_{\mathrm{w}}-\mathrm{SO}_{3}$ and MSNs-Phenyl, respectively). All nanoplatforms were characterized showing average size from 100-150 $\mathrm{nm}$ and negative surface charge $(\sim$ $40 \mathrm{mV}$ ), conferring good colloidal stability by interparticle repulsion. Antiviral in vitro assays toward HSV-1 and HSV-2 were carried out displaying that sulfonate group presence was as a prerequisite for antiviral activity, with the benzene group (from benzene sulfonate-modified MSNs) enhancing the antiviral response regarding to an alkyl group (propyl sulfonatemodified MSNs). Phenyl-modified MSNs showed a relatively weak antiviral activity, reinforcing the sulfonate group importance. From second study concerning ACV-loaded benzene sulfonate-modified MSNs (labeled ACV@MSNs-phenyl$\left.\mathrm{SO}_{3}\right)$, high drug loading $(34 \% \mathrm{w} / \mathrm{w})$ and controlled and prolonged ACV release $(70 \%(\mathrm{w} / \mathrm{w})$ up to $24 \mathrm{~h})$ were detected. This release profile can be assigned to the mesopores functionalization with hydrophobic groups which can act as blockers controlling drug release. To evaluate post viral penetration into cells, Vero cells were infected with HSV-1 and then, treated with the nanoplatforms. Results exhibited higher antiviral activity for ACV@MSNs-phenyl-SO 3 than ACV@MSNs and free ACV. The targeting effect of GAG mimetic from ACV@MSNs-phenyl-SO $\mathrm{S}_{3}$ against HSV-1 was investigated achieving 100\% antiviral activity whereas both no interaction with HSV-1 were detected for ACV@MSNs and free ACV. These results confirmed that the GAG mimetic-functionalized MSNs performed dual action mechanism against HSV-1 and HSV-2 opening up new possibilities for virus infections treatment.

As aforementioned, viruses comprise a harmful pathogen to the humans responsible for millions of people death every year. Despites some therapies such as highly active antiretroviral therapy (HAART) available to treat human immunodeficiency virus (HIV), the demand for new therapies and vaccines are essential to fight against new viruses. To better understand the mechanism of antiviral MSNs action, Silva et al . (54) developed modified MSNs to evaluate their biocompatibility and their ability to inhibit the virus transduction of target cells. (3-aminopropyl)triethoxysilane (APTES), (3-glycidyloxypropyl)trimethoxysilane (GPTMS) and trimethoxy-(2phenylethyl)silane (TMPES) groups were used to modified
MSNs surface and noted as MSNs-APTES, MSNs-GPTMS and MSNs-TMPES. Spherical-shaped modified MSNs with average size from 354-571 nm and negative surface charge (from -11.2 to $-22.8 \mathrm{mV}$ ) were fabricated. Cytotoxicity assays were performed for the nanosystems exhibiting cells metabolic activity similar to the control at lower particle concentration $\left(10^{-5} \mathrm{~g} \mathrm{~mL}^{-1}\right)$ and cells nuclei morphology preserved after interaction. The inhibitory potential of the nanosystems to prevent virus transduction was evaluated by using a truncated version of the HIV-gp120 glycoprotein that can interact with cells containing CD4 receptor and VSV-G envelope that can bind on different cell surfaces. When target cell was transduced by virus, GFP is expressed by the infected cell, that means, whether transduction occurs GFP can be detected. In this way, the pseudotyped preparation was treated with the nanosystems. At low concentration, all nanosystems reduced transduction for both recombinant lentivirus with VSV-G and HIV-gp120 envelopes. MSNs-APTES and MSNs-TMPES showed higher transduction reduction for VSV-G $(\sim 50 \%)$ and HIV-gp $120(\sim 20 \%)$ envelopes, respectively. Theoretical studies suggested that higher hydrophobicity could favor interaction with HIV-gp120 whereas hydrophilicity could lead to better interaction with VSV-G, explain the distinct responses. These results provide new insights towards the design of specific MSNs-based nanocarriers as new strategies for viral control and anti-HIV therapy.

Porcine circovirus type 2 (PCV2) is a primary etiological agent of post-weaning multi-systemic wasting syndrome (PMWS). PMWS are severe in acute outbreaks, causing death of up to $80 \%$ of affected animals. To develop new therapies for PGV2 infections is essential for pig industries. For this reason, Guo et al . (148) reported the preparation of PCV2 GST-ORF2-E protein-loaded HMSNs as nanovaccines aiming to induce persistent immune responses of PCV2. The nanosystem was characterized exhibiting spherical shape with average size of $200 \mathrm{~nm}$. Maximum protein loading amount of $15 \%(\mathrm{w} / \mathrm{w})$ were achieved. Protein release profile (PBS at $\mathrm{pH}$ 7 ) was time-dependent showing rapid release $(\sim 50 \%)$ up to $12 \mathrm{~h}$ and slow release up to the sixth day. To evaluate the specificity of mice antibodies immunized by GST-ORF2-E, mouse sera were used in immunofluorescence experiments to determine the specificity of antibodies by PCV2-infected PK15 cells. Results displayed that no significant staining was detected suggesting specificity of the mouse antibody against PGV2. Indirect ELISA assays displayed that the antibody titers of mice immunized with PGV2 GST-ORF2E@HMSNs were higher than those of mice immunized with the GST-ORF2-E protein, especially at the third- and fourthweeks post-vaccination. Furthermore, the T-lymphocyte proliferation response in mice induced by the PGV2 GST-ORF2E@HMSNs remained at levels higher than those in mice immunized with the GST-ORF2-E protein, demonstrating that the proteins loaded into the HMSNs not only stimulate 
humoral and cellular immune responses but also induce persistent immune responses because of the release kinetics from HMSNs. Results confirmed HMSNs as an interesting an efficient vaccine nanovehicle for PCV2 treatment and further studies regarding virus therapy.

\section{CLINICAL TRANSLATION, CHALLENGES AND FUTURE OUTLOOK}

The challenges of any designed nanostructure for therapeutic purposes consists on achieve good biocompatibility, biodegradability, complete and short clearance time as already stated by US FDA. Considering the aforementioned, MSNs are very attractive as nanocarriers/nanotherapeutics because their interesting properties such as chemical and physical stabilities, size, morphology, surface and pores structures can be easily modulated to achieve good biological behavior and also effective drug loading and delivery at the desired site, comprising efficient nanoplatforms in most of pre-clinical assays. Internal, external or multi stimuli-responsive MSNs controlled drug delivery systems have been designed as smart nanoarchitectures that control pharmacokinetics parameters and emphasize the amazing properties of MSNs, stimulating researches towards their application in clinical trials $(12,160,161)$.

Wiesner group $(162,163)$ described the fabrication of "Cornell dots or C dots" (labeled ${ }^{124}$ IcRGDY-PEG-C dots) as the first nanosilica approved by the FDA for clinical assays. A smart core-shell nanosilica containing Cy5 dye and ${ }^{124} \mathrm{I}$ radiolabels for optical-PET (positron emission tomography) dual imaging was fabricated and modified with PEG and cRGDY peptides as target agent for molecular expression in human melanoma xenografts $(162,163)$. Biological behavior was evaluated showing fast Cornell dots ( $\mathrm{C}$ dots) excretion via the kidneys $(\sim 90 \%)$ and hepatobiliary route $(\sim 10 \%)$ with excretion times from 13 to $21 \mathrm{~h}$ (163). These smart silicabased nanostructures were successfully applied as nanoprobes for tumors detection and monitoring in clinical assays (163-165).

Chen et al . (166) described the fabrication of $\mathrm{C}$ dots conjugated with the radiometal Zirconium-89 $\left({ }^{89} \mathrm{Zr}, \mathrm{tl} / 2=78.4\right.$ h) using two different strategies: chelator-free and chelatorbased radiolabeling. The best nanosystem (labeled ${ }^{89} \mathrm{Zr}$ DFO-cRGDY-PEG-C' dots) fabricated by chelator-based radiolabeling exhibited higher in vivo radiostability with good renal clearance, low reticuloendothelial system (RES) accumulation, high tumor uptake and target-to-background ratios relative to biological controls for human melanoma xenograft models. The first clinical trial using ${ }^{89} \mathrm{Zr-DFO-cRGDY-PEG-}$ $\mathrm{C}^{\prime}$ dots has been carried out for bioimaging of malignant brain tumors (NCT03465618).
Kharlamov et al . (167) described the application of plasmonic photothermal therapy (PPTT) by using near-infrared (NIR) laser irradiation onto silica-gold nanoparticles (AuNPs) for Angioplasty and artery remodeling. Interesting safety level and significant treatment efficacy with plaque burden reduction $\left(79.4 \mathrm{~mm}^{3}\right)$ was successfully achieved. In this regard, authors (168) conducted a first-in-man trial (labeled NANOM-FIM, NCT01270139) by using multifunctional silica-AuNPs-dispersed on-artery patch associated with PPTT for atherosclerosis treatment. Results exhibited significant regression of coronary atherosclerosis with good biocompatibility and no significant toxicity besides lower risk of cardiovascular death in humans. This bioengineered nanostructure offers great potential for further atherosclerosis theranostic purposes.

The clinical applications of aforementioned silica-based nanostructures encourage researchers focuses on MSNs clinical translation and further commercialization. Although several researches have been performed in the last two decades towards the biological behavior and preclinical evaluation of smart and engineered stimuli-responsive MSNs for monitoring and/or therapeutic purposes (12,88,89,96,161,169-171), achieve their clinical translation is still a great challenge requiring long-term stability, scale-up of these nanostructures (from bench to industrial scale-up) and overcome obstacles in animal trials and regulating authorities evaluation (160,161,171). Despites the numerously reports concerning multifunctional MSNs fabrication, their scale-up is rarely described. Some authors $(172,173)$ reported large-scale synthesis of dense and hollow mesoporous silica micro/nanoparticles (173) and monodisperse small MSNs with a fine size distribution at room temperature $\left(27^{\circ} \mathrm{C}\right)$ (172), both methods using CTAB as template. The structural stability of nanoparticles is usually determined by interaction with the biological environment and between themselves. Poor colloidal and thermal stabilities can result in drawbacks with long-term storage and nanosystems administration. In this sense, MSNs present robust silica framework and exceptional electronic architecture easily optimized, making them amazing nanoarchitectures with interesting structural stability for biomedical applications (174). Additionally, authors reported that the association of MSNs with metals such as iron, $\mathrm{Pt}, \mathrm{Al}$ and others, can improve the nanoplatforms thermal and physical stabilities considering a critical ratio of metal to silica content (174).

Up to now, preclinical assays related to MSNs were successfully performed whereas no MSNs have been described in clinical trials. Authors (160) suggested that the clinical translation has failed due to the large physiological distinction between small animals and humans, indicating that animal models are not the better indication for further nanostructures success in humans. In addition, authors suggest that rather 
than prolonged xenografting experiments (in cancer treatment), positive animal toxicological results could allow to start phase 1 of clinical trials.

Another concern related to MSNs or any nanostructures for clinical translation relies on regulatory agencies (as US FDA) evaluation. Currently, nanostructured materials are evaluated as small-molecule drugs, being that some nanosystems can act only as nanoprobes or without loaded drugs. In this sense, the actual evaluation processes by agencies comprise a bottleneck that allow only a few percentages of nanostructures can be approved for commercial purposes. Authors suggested that regulatory agencies could create specific evaluation category for nanomedicines aiming to streamline their translation $(160,161,171)$.

Finally, taking into account all abovementioned challenges, the tunable properties of MSNs and recent advances regarding smart and multi stimuli-responsive MSNs for diagnosis, therapy or theranostic applications, we can expect in the next years MSNs-based nanoplatforms and other silica-based nanosystems will become approved by FDA, impacting the treatment way and global human health.

\section{CONCLUSIONS}

Currently, there is a great global concern regarding infectious diseases, especially those without well-established therapy or, mostly, which exhibits multidrug resistant microorganisms. Recently, nanomedicine has emerged from the nanotechnology as a wide and great approach to overcome drawbacks in biomedical field imposed by several diseases. More precisely, MSNs have great potential for biomedical application due to their interesting properties including biocompatibility, biodegradability, easy clearance, tunable physicochemical properties as size, shape and pores size. MSNs multifunctionality opens up several possibilities to fabricate smart and targeted nanomedicines or nanovaccines for infectious diseases prevention and treatment. These nanoplatforms can promote specific and controlled drug release at target disease cells/tissues improving treatment efficacy minimizing side effects besides no patient compliance. Despites MSNs potential has received great attention in the last years towards the design of new formulations and therapies, MSNs applications to treat infectious diseases such as parasites, viruses and fungi have been already scarce up to now. As depicted in this review, only MSNs-based nanocarriers for bacterial infections and treatment have been widely exploited, which opens up several opportunities to design multifunctional MSNs-based nanoplatforms for controlled drug delivery to threat the most of infectious diseases presented nowadays. Although very few reports are described regarding MSNs-based nanovehicles in clinical assays, the good biological behavior, unceasing preclinical tests performed and the last trends using these nanosystems, allow us to prospect a clinical translation in a near future.

ACKNOWLEDGMENTS AND DISCLOSURES. This study was financed in part by the Coordenação de Aperfeiçoamento de Pessoal de Ensino Superior - Brasil (CAPES) - Finance code - 001. The authors also are grateful to the São Paulo Research Foundation (FAPESP, Brazil, Grant \#2018/253773) for research fellowship and financial support. This study is part of the National Institute of Science and Technology in Pharmaceutical Nanotechnology: a transdisciplinary approach INCT-NANOFARMA, which is supported by São Paulo Research Foundation (FAPESP, Brazil) Grant \#2014/50928-2, and by "Conselho Nacional de Desenvolvimento Científico e Tecnológico" (CNPq, Brazil) Grant \# 465687/2014-8. The authors report no conflicts of interest associated with this publication.

Publisher's Note Springer Nature remains neutral with regard to jurisdictional claims in published maps and institutional affliations.

\section{REFERENCES}

1. Ogunsona EO, Muthuraj R, Ojogbo E, Valerio O, Mekonnen TH. Engineered nanomaterials for antimicrobial applications: A review. Appl Mater Today. 2020;18:100473 https://linkinghub. elsevier.com/retrieve/pii/S235294071930592X.

2. Zaidi S, Misba L, Khan AU. Nano-therapeutics: A revolution in infection control in post antibiotic era. Nanomedicine Nanotechnology, Biol Med. 2017;13(7):2281-301 https:// linkinghub.elsevier.com/retrieve/pii/S1549963417301235.

3. WHO|Antimicrobial resistance.; 2020 Jun 2. Available from: https://www.who.int/news-room/fact-sheets/detail/ antimicrobial-resistance

4. Lee N-Y, Ko W-G, Hsueh P-R. Nanoparticles in the treatment of infections caused by multidrug-resistant organisms. Front Pharmacol. 2019;10 https://www.frontiersin.org/article/10. 3389/fphar.2019.01153/full.

5. Chen Y-C, Chen S-J, Cheng H-F, Yeh M-K. Development of Yersinia pestis $\mathrm{F} 1$ antigen-loaded liposome vaccine against plague using microneedles as a delivery system. J Drug Deliv Sci Technol. 2020;55:101443 https://linkinghub.elsevier.com/retrieve/pii/ S1773224719314820.

6. Weiss C, Carriere M, Fusco L, Capua I, Regla-Nava JA, Pasquali $\mathrm{M}$, et al. Toward nanotechnology-enabled approaches against the COVID-19 pandemic. ACS Nano. 2020;14(6):6383-406. https://doi.org/10.1021/acsnano.0c03697.

7. Emerich DF, Thanos CG. Nanotechnology and medicine. Expert Opin Biol Ther. 2003;3(4):655-63. https://doi.org/10.1517/ 147 12598.3.4.655.

8. Vallet-Regí M, González B, Izquierdo-Barba I. Nanomaterials as promising alternative in the infection treatment. Int $\mathrm{J}$ Mol Sci. 2019;20(15):3806 https://www.mdpi.com/1422-0067/20/15/ 3806.

9. Singh M, Singh S, Prasad S, Gambhir IS. Nanotechnology in medicine and antibacterial effect of silver nanoparticles. Vol. 3, Digest Journal of Nanomaterials and Biostructures. 2008. 
10. Smerkova K, Dolezelikova K, Bozdechova L, Heger Z, Zurek L, Adam V. Nanomaterials with active targeting as advanced antimicrobials. WIREs Nanomedicine and Nanobiotechnology. 2020;el636. https://doi.org/10.1002/wnan.1636

11. Pallotta A, Clarot I, Sobocinski J, Fattal E, Boudier A. Nanotechnologies for medical devices: potentialities and risks. ACS Appl Bio Mater. 2019;2(1):1-13. https://doi.org/10. 1021/acsabm.8b00612.

12. Sábio RM, Meneguin AB, Ribeiro TC, Silva RR, Chorilli M. New insights towards mesoporous silica nanoparticles as a technological platform for chemotherapeutic drugs delivery. Int J Pharm. 2019;564:379-409 https://linkinghub.elsevier.com/retrieve/pii/ S0378517319303308.

13. Drouet G. Nanotechnologies: A key role in virus fight. Biomed J Sci Tech Res. 2020;27(2):20774-82.

14. Li A, Long L, Liu F, Liu J, Wu X, Ji Y. Antigen-labeled mesoporous silica-coated $\mathrm{Au}$-core Pt-shell nanostructure: a novel nanoprobe for highly efficient virus diagnosis. J Biol Eng. 2019;13(1):87 https://jbioleng.biomedcentral.com/articles/10.1186/s13036019-0220-1.

15. Bonifácio B, Silva P, Ramos M, Negri K, Maria Bauab T, Chorilli M. Nanotechnology-based drug delivery systems and herbal medicines: a review. Int J Nanomedicine. 2013;9(1):1 http://www. dovepress.com/nanotechnology-based-drug-delivery-systemsand-herbal-medicines-a-revi-peer-reviewed-article-IJN .

16. Calixto G, Bernegossi J, de Freitas L, Fontana G, Chorilli M. Nanotechnology-based drug delivery systems for photodynamic therapy of cancer: A review. Molecules. 2016;21(3):342 http:// www.mdpi.com/1420-3049/21/3/342.

17. Nazemiyeh E, Eskandani M, Sheikhloie H, Nazemiyeh H. Formulation and physicochemical characterization of lycopeneloaded solid lipid nanoparticles. Adv Pharm Bull. 2016;6(2):23541 http://journals.tbzmed.ac.ir/APB/Abstract/APB_2512_ 20160131131808

18. Sahoo S, Dilnawaz F, Krishnakumar S. Nanotechnology in ocular drug delivery. Drug Discov Today. 2008;13(3-4):144-51 https:// linkinghub.elsevier.com/retrieve/pii/S1359644607004618.

19. Arriagada F, Nonell S, Morales J. Silica-based nanosystems for therapeutic applications in the skin. Nanomedicine. 2019;14(16): 2243-67. https://doi.org/10.2217/nnm-2019-0052.

20. Sahoo SK, Labhasetwar V. Nanotech approaches to drug delivery and imaging. Drug Discov Today. 2003;8(24):1112-20 https:// linkinghub.elsevier.com/retrieve/pii/S1359644603029039.

21. Madhusoodana CD, Kameshima Y, Nakajima A, Okada K, Kogure T, MacKenzie KJD. Synthesis of high surface area Alcontaining mesoporous silica from calcined and acid leached kaolinites as the precursors. J Colloid Interface Sci. 2006;297(2):724 31 https://linkinghub.elsevier.com/retrieve/pii/ S002197970501088X.

22. Yokoi T, Kubota Y, Tatsumi T. Amino-functionalized mesoporous silica as base catalyst and adsorbent. Appl Catal A Gen. 2012;421-422:14-37 https://linkinghub.elsevier.com/retrieve/ pii/S0926860X12000646.

23. Chen W, Glackin CA, Horwitz MA, Zink JI. Nanomachines and other caps on mesoporous silica nanoparticles for drug delivery. Acc Chem Res. 2019;52(6):1531-42. https://doi.org/10.1021/ acs.accounts.9b00116.

24. Manzano M, Vallet-Regí M. Mesoporous silica nanoparticles for drug delivery. Adv Funct Mater. 2020;30(2):1902634. https:// doi.org/10.1002/adfm.201902634.

25. Mohammadpour R, Cheney DL, Grunberger JW, Yazdimamaghani M, Jedrzkiewicz J, Isaacson KJ, et al. Oneyear chronic toxicity evaluation of single dose intravenously administered silica nanoparticles in mice and their Ex vivo human hemocompatibility. J Control Release. 2020;324:471-81 https://linkinghub.elsevier.com/retrieve/pii/ S0168365920303047.

26. Sábio RM, Santagneli SH, Gressier M, Gaiut JMA, Pazin WM, Leite IS, et al. Luminescent nanohybrids based on silica and silylated $\mathrm{Ru}(\mathrm{II})-\mathrm{Yb}(\mathrm{III})$ heterobinuclear complex: new tools for biological media analysis. Nanotechnology. 2020;31(8):085709 https://iopscience.iop.org/article/10.1088/1361-6528/ab55c3.

27. Sábio RM, Santagneli SH, Gressier M, Caiut JMA, Pazin WM, Ribeiro SJL, et al. Near-infrared/visible-emitting nanosilica modified with silylated $\mathrm{Ru}(\mathrm{II})$ and $\mathrm{Ln}(\mathrm{III})$ complexes. Nanotechnology. 2020;31(3):035602 https://iopscience.iop.org/article/10.1088/ 1361-6528/ab494f.

28. Baeza A, Ruiz-Molina D, Vallet-Regí M. Recent advances in porous nanoparticles for drug delivery in antitumoral applications: inorganic nanoparticles and nanoscale metal-organic frameworks. Expert Opin Drug Deliv. 2017;14(6):783-96. https://doi. org/10.1080/17425247.2016.1229298.

29. Lu J, Liong M, Li Z, Zink JI, Tamanoi F. Biocompatibility, biodistribution, and drug-delivery efficiency of mesoporous silica nanoparticles for cancer therapy in animals. Small. 2010;6(16): 1794-805. https://doi.org/10.1002/smll.201000538.

30. National Institutes of Health. Understanding emerging and reemerging infectious diseases. Biol Sci Curric study NIH Curric Suppl Ser Natl Institutes Heal Bethesda, MD. 2007.

31. Guido RVC, Andricopulo AD, Oliva G. Planejamento de fármacos, biotecnologia e química medicinal: aplicações em doenças infecciosas. Estud Avançados. 2010;24(70):81-98 http://www.scielo.br/scielo.php?script=sci arttext\&pid=S0103$40142010000300006 \& \operatorname{lng}=$ pt\&tlng $=$ pt.

32. WHO | Infectious diseases.; 2020 May 22. Available from: https://www.who.int/topics/infectious diseases/en/

33. Rohr JR, Barrett CB, Civitello DJ, Craft ME, Delius B, DeLeo GA, et al. Emerging human infectious diseases and the links to global food production. Nat Sustain. 2019;2(6):445-56 http:// www.nature.com/articles/s41893-019-0293-3.

34. WHO | Disease outbreaks.; 2020 May 22. Available from: https://www.who.int/emergencies/diseases/en/

35. Li G, Fan Y, Lai Y, Han T, Li Z, Zhou P, et al. Coronavirus infections and immune responses. J Med Virol. 2020;92(4):424 32. https://doi.org/10.1002/jmv.25685.

36. Fujii Y, Goto H, Watanabe T, Yoshida T, Kawaoka Y. Selective incorporation of influenza virus RNA segments into virions. Proc Natl Acad Sci. 2003;100(4):2002-7. https://doi.org/10.1073/ pnas.0437772100.

37. Sissoko D, Duraffour S, Kerber R, Kolie JS, Beavogui AH, Camara A-M, et al. Persistence and clearance of Ebola virus RNA from seminal fluid of Ebola virus disease survivors: a longitudinal analysis and modelling study. Lancet Glob Heal. 2017;5(1):e80-8 https://linkinghub.elsevier.com/retrieve/pii/ S2214109X16302431.

38. Kitamura N, Semler BL, Rothberg PG, Larsen GR, Adler CJ, Dorner AJ, et al. Primary structure, gene organization and polypeptide expression of poliovirus RNA. Nature. 1981;291(5816): 547-53 http://www.nature.com/articles/291547a0.

39. Arroyo J, Miller CA, Catalan J, Monath TP. Yellow fever vector live-virus vaccines: West Nile virus vaccine development. Trends Mol Med. 2001;7(8):350-4 https://linkinghub.elsevier.com/ retrieve/pii/S1471491401020482.

40. Barrett ADT, Higgs S. Yellow fever: A disease that has yet to be conquered. Annu Rev Entomol. 2007;52(1):209-29. https://doi. org/10.1146/annurev.ento.52.110405.091454.

41. Poddar SK. Influenza virus types and subtypes detection by single step single tube multiplex reverse transcription-polymerase chain reaction (RT-PCR) and agarose gel electrophoresis. J Virol Methods. 2002;99(1-2):63-70 https://linkinghub.elsevier.com/ retrieve/pii/S0166093401003809. 
42. Tatara AM. Role of tissue engineering in COVID-19 and future viral outbreaks. Tissue Eng Part A. 2020;26(9-10):468-74. https://doi.org/10.1089/ten.tea.2020.0094.

43. Zhu N, Zhang D, Wang W, Li X, Yang B, Song J, et al. A novel coronavirus from patients with pneumonia in China, 2019. N Engl J Med. 2020;382(8):727-33. https://doi.org/10.1056/ NEJMoa2001017.

44. Feldmann H, Geisbert TW. Ebola haemorrhagic fever. Lancet. 2011;377(9768):849-62 https://linkinghub.elsevier.com/ retrieve/pii/S0140673610606678.

45. Tuboi SH, Costa ZGA, da Costa Vasconcelos PF, Hatch D. Clinical and epidemiological characteristics of yellow fever in Brazil: analysis of reported cases 1998-2002. Trans R Soc Trop Med Hyg. 2007;101(2):169-75. https://doi.org/10.1016/j. trstmh.2006.04.001.

46. Monath TP, Vasconcelos PFC. Yellow fever. J Clin Virol. 2015;64:160-73 https://linkinghub.elsevier.com/retrieve/pii/ S1386653214003692.

47. Tumpey TM. Characterization of the reconstructed 1918 Spanish Influenza pandemic virus. Science. 2005;310(5745):77-80. https://doi.org/10.1126/science.1119392.

48. Heikkinen LS, Kazlauskas A, Melén K, Wagner R, Ziegler T, Julkunen I, et al. Avian and 1918 Spanish Influenza A virus NS 1 proteins bind to Crk/CrkL Src homology 3 domains to activate host cell signaling. J Biol Chem. 2008;283(9):5719-27. https:// doi.org/10.1074/jbc.M707 195200.

49. Henry R. Etymologia: poliomyelitis. Emerg Infect Dis. 2019;25(8): 1611-1 http://wwwnc.cdc.gov/eid/article/25/8/ET-2508 article.htm

50. Lahiri BB, Divya MP, Bagavathiappan S, Thomas S, Philip J. Detection of pathogenic gram negative bacteria using infrared thermography. Infrared Phys Technol. 2012;55(6):485-90 https:/ / linkinghub.elsevier.com/retrieve/pii/ S1350449512000692.

51. Deen J, Mengel MA, Clemens JD. Epidemiology of cholera. Vaccine. 2020;38:A31-40 https://linkinghub.elsevier.com/ retrieve/pii/S0264410X19309995

52. Koelman DLH, Brouwer MC, van de Beek D. Targeting the complement system in bacterial meningitis. Brain. 2019;142(11): 3325-37 https://academic.oup.com/brain/article-abstract/ $142 / 11 / 3325 / 5543074$.

53. Epstein FH, Quagliarello V, Scheld WM. Bacterial meningitis: Pathogenesis, pathophysiology, and progress. N Engl J Med. 1992;327(12):864-72. https://doi.org/10.1056/ NEJM199209173271208.

54. de Souza e Silva JM, Hanchuk TDM, Santos MI, Kobarg J, Bajgelman MC, Cardoso MB. Viral inhibition mechanism mediated by surface-modified silica nanoparticles. ACS Appl Mater Interfaces. 2016;8(26):16564-16572. https://doi.org/10.1021/ acsami.6b03342

55. De Lyra MAM, Soares-Sobrinho JL, Brasileiro MT, De MF, Roca L, Barraza JA, et al. Sistemas matriciais hidrofilicos e mucoadesivos para liberação controlada de fármacos. Lat Am J Pharm. 2007;26(5):784-93.

56. Momčilović S, Cantacessi C, Arsić-Arsenijević V, Otranto D, Tasić-Otašević S. Rapid diagnosis of parasitic diseases: current scenario and future needs. Clin Microbiol Infect. 2019;25(3): 290-309 https://linkinghub.elsevier.com/retrieve/pii/ S1198743X18303951.

57. Hobbs EC, Trevisan C, Johansen MV, Dorny P, Gabriël S. Value of electronic educational media in combatting parasitic diseases. Trends Parasitol. 2019;35(3):173-6 https://linkinghub.elsevier. com/retrieve/pii/S1471492218302186.

58. Figueiras ARR, Coimbra AB, Veiga FJB. Nanotecnologia na saúde: aplicações e perspectivas. Bol Inf Geum. 2014;5(2):14-26
https://www.academia.edu/25482960/Nanotecnologia_na_ saúde aplicações e perspectivas.

59. Ferreira Soares DC, Soares LM, Miranda de Goes A, Melo EM, Branco de Barros AL, Alves Santos Bicalho TC, et al. Mesoporous SBA-16 silica nanoparticles as a potential vaccine adjuvant against Paracoccidioides brasiliensis. Microporous Mesoporous Mater. 2020;291:109676. https://linkinghub.elsevier.com/retrieve/pii/ S1387181119305335

60. Edrissian G, Rokni M, Mohebali M. History of medical parasitology and parasitic infections in Iran. Archives of iranian medicine. 2016;19(8):601-7 http://www.ams.ac.ir/AIM/NEWPUB/16/ 19/8/0014.pdf.

61. Narayan R, Nayak U, Raichur A, Garg S. Mesoporous silica nanoparticles: a comprehensive review on synthesis and recent advances. Pharmaceutics. 2018;10(3):118 http://www.mdpi. com/1999-4923/10/3/118.

62. Trewyn BG, Slowing II, Giri S, Chen H-T, Lin VSY. Synthesis and functionalization of a mesoporous silica nanoparticle based on the sol-gel process and applications in controlled release. Acc Chem Res. 2007;40(9):846-53. https://doi.org/10.1021/ ar600032u.

63. Øye G, Sjöblom J, Stöcker M. Synthesis, characterization and potential applications of new materials in the mesoporous range. Adv Colloid Interface Sci. 2001;89-90:439-66 https:// linkinghub.elsevier.com/retrieve/pii/S000186860000066X.

64. Tozuka Y, Wongmekiat A, Kimura K, Moribe K, Yamamura S, Yamamoto K. Effect of pore size of FSM-16 on the entrapment of flurbiprofen in mesoporous structures. Chem Pharm Bul. 2005;53(8):974-7 http://joi.jlc.jst.go.jp/JST.JSTAGE/cpb/53. 974?from $=$ CrossRef.

65. Nandiyanto ABD, Kim S-G, Iskandar F, Okuyama K. Synthesis of spherical mesoporous silica nanoparticles with nanometer-size controllable pores and outer diameters. Microporous Mesoporous Mater. 2009;120(3):447-53 https://linkinghub.elsevier.com/ retrieve/pii/S1387181108006495.

66. Kumar D, Schumacher K, du Fresne von Hohenesche C, Grün M, Unger K. MCM-41, MCM-48 and related mesoporous adsorbents: their synthesis and characterisation. Colloids Surfaces A Physicochem Eng Asp. 2001;187-188:109-16 https: / / linkinghub.elsevier.com/retrieve/pii / S0927775701006380.

67. Ukmar T, Planinšek O. Ordered mesoporous silicates as matrices for controlled release of drugs. Acta Pharm. 2010;60(4):373-85 http://content.sciendo.com/view/journals/acph/60/4/articlep373.xml.

68. Wang S, Li H. Structure directed reversible adsorption of organic dye on mesoporous silica in aqueous solution. Microporous Mesoporous Mater. 2006;97(1-3):21-6 https://linkinghub. elsevier.com/retrieve/pii/S1387181106003076.

69. Kleitz F, Liu D, Anilkumar GM, Park I-S, Solovyov LA, Shmakov $\mathrm{AN}$, et al. Large cage face-centered-cubic Fm 3 m mesoporous silica: synthesis and structure. J Phys Chem B. 2003;107(51): 14296-300. https://doi.org/10.1021/jp036136b.

70. Jammaer J, Aerts A, D'Haen J, Seo JW, Martens JA. Convenient synthesis of ordered mesoporous silica at room temperature and quasi-neutral pH. J Mater Chem. 2009;19(44):8290 http://xlink. rsc.org/?DOI=b915273c.

71. Gao C, Qiu H, Zeng W, Sakamoto Y, Terasaki O, Sakamoto K, et al. Formation mechanism of anionic surfactant-templated mesoporous silica. Chem Mater. 2006;18:3904-14. https://doi.org/ $10.1021 / \mathrm{cm} 061107 \% 2 \mathrm{~B}$.

72. Grün M, Lauer I, Unger KK. The synthesis of micrometer- and submicrometer-size spheres of ordered mesoporous oxide MCM41. Adv Mater. 1997;9(3):254-7. https://doi.org/10.1002/adma. 19970090317. 
73. Stöber W, Fink A, Bohn E. Controlled growth of monodisperse silica spheres in the micron size range. J Colloid Interface Sci. 1968;26(1):62-9 https://linkinghub.elsevier.com/retrieve/pii/ 0021979768902725 .

74. Flodström K, Wennerström H, Alfredsson V. Mechanism of mesoporous silica formation. A time-resolved NMR and TEM study of silica-block copolymer aggregation. Langmuir. 2004;20(3): 680-8. https://doi.org/10.1021/la030173c.

75. Hollamby MJ, Borisova D, Brown P, Eastoe J, Grillo I, Shchukin D. Growth of mesoporous silica nanoparticles monitored by timeresolved small-angle neutron scattering. Langmuir. 2012;28(9): 4425-33. https://doi.org/10.1021/la203097x.

76. Edler KJ. Current understanding of formation mechanisms in surfactant-templated materials. Aust J Chem. 2005;58(9):627 http://www.publish.csiro.au/?paper=CH05141.

77. Yang P, Gai S, Lin J. Functionalized mesoporous silica materials for controlled drug delivery. Chem Soc Rev. 2012;41(9):3679-98. ttps://pubs.rsc.org/en/content/articlehtml/2012/cs/ c2cs15308d

78. Qu F, Zhu G, Lin H, Zhang W, Sun J, Li S, et al. A controlled release of ibuprofen by systematically tailoring the morphology of mesoporous silica materials. J Solid State Chem. 2006;179(7): 2027-35.

79. Heikkilä T, Salonen J, Tuura J, Hamdy M, Mul G, Kumar N, et al. Mesoporous silica material TUD-1 as a drug delivery system. Int J Pharm. 2007;331(1):133-8 https://linkinghub.elsevier.com/ retrieve/pii/S0378517306007708.

80. Heikkilä T, Salonen J, Tuura J, Kumar N, Salmi T, Murzin DY, et al. Evaluation of mesoporous TCPSi, MCM-41, SBA-15, and TUD-1 materials as API carriers for oral drug delivery. Drug Deliv. 2007;14(6):337-47. https://doi.org/10.1080/ 10717540601098823.

81. Andersson J, Rosenholm J, Areva S, Lindén M. Influences of material characteristics on ibuprofen drug loading and release profiles from ordered micro- and mesoporous silica matrices. Chem Mater. 2004;16(21):4160-7 https://pubs.acs.org/ sharingguidelines.

82. Vallet-Regí M. Ordered mesoporous materials in the context of drug delivery systems and bone tissue engineering. Chem - A Eur J. 2006;12(23):5934-43. https://doi.org/10.1002/chem. 200600226

83. Vallet-Regi M, Balas F, Colilla M, Manzano M. Drug confinement and delivery in ceramic implants. Drug Metab Lett. 2007;1(1):37-40 http://www.eurekaselect.com/openurl/content. php? genre $=$ article $\&$ issn $=1872-3128 \&$ volume $=1 \&$ issue $=$ 1 \&spage $=37$.

84. Vallet-Regí M, Balas F, Arcos D. Mesoporous materials for drug delivery. Angew Chemie Int Ed. 2007;46(40):7548-58. https:// doi.org/10.1002/anie.200604488.

85. Huang X, Li L, Liu T, Hao N, Liu H, Chen D, et al. The shape effect of mesoporous silica nanoparticles on biodistribution, clearance, and biocompatibility in vivo. ACS Nano. 201 1;5(7):5390-9 www.acsnano.org.

86. Huang X, Teng X, Chen D, Tang F, He J. The effect of the shape of mesoporous silica nanoparticles on cellular uptake and cell function. Biomaterials. 2010;31(3):438-48 https://linkinghub. elsevier.com/retrieve/pii/S0142961209009922.

87. Tang F, Li L, Chen D. Mesoporous silica nanoparticles: synthesis, biocompatibility and drug delivery. Adv Mater. 2012;24(12): 1504-34. https://doi.org/10.1002/adma.201104763.

88. Croissant JG, Fatieiev Y, Almalik A, Khashab NM. Mesoporous Silica and organosilica nanoparticles: physical chemistry, biosafety, delivery strategies, and biomedical applications. Adv Healthc Mater. 2018;7(4):1700831. https://doi.org/10.1002/adhm. 201700831
89. Yang B, Chen Y, Shi J. Mesoporous silica/organosilica nanoparticles: Synthesis, biological effect and biomedical application. Mater Sci Eng R Reports. 2019;137:66-105 https://linkinghub. elsevier.com/retrieve/pii/S0927796X18302328.

90. Giret S, Wong Chi Man M, Carcel C. Mesoporous-silicafunctionalized nanoparticles for drug delivery. Chem - A Eur J. 2015;2 1(40):13850-65. https://doi.org/10.1002/chem. 201500578.

91. Slowing II, Wu C-W, Vivero-Escoto JL, Lin VS-Y. Mesoporous silica nanoparticles for reducing hemolytic activity towards mammalian red blood cells. Small. 2009;5(1):57-62. https://doi.org/ 10.1002/smll.200800926.

92. Nash T, Allison AC, Harington JS. Physico-chemical properties of silica in relation to its toxicity. Nature. 1966;210(5033):259-61 http://www.nature.com/articles/210259a0.

93. Lin Y-S, Haynes CL. Impacts of mesoporous silica nanoparticle size, pore ordering, and pore integrity on hemolytic activity. J Am Chem Soc. 2010;132(13):4834-42. https://doi.org/10.1021/ ja910846q

94. Liu T, Li L, Teng X, Huang X, Liu H, Chen D, et al. Single and repeated dose toxicity of mesoporous hollow silica nanoparticles in intravenously exposed mice. Biomaterials. 2011;32(6):1657-68 https:/ / linkinghub.elsevier.com/retrieve/pii/ S0142961210013426.

95. Fu C, Liu T, Li L, Liu H, Chen D, Tang F. The absorption, distribution, excretion and toxicity of mesoporous silica nanoparticles in mice following different exposure routes. Biomaterials. 2013;34(10):2565-75 https://linkinghub.elsevier.com/retrieve/ pii/S0142961213000082.

96. Croissant JG, Fatieiev Y, Khashab NM. Degradability and clearance of silicon, organosilica, silsesquioxane, silica mixed oxide, and mesoporous silica nanoparticles. Adv Mater. 2017;29(9): 1604634. https://doi.org/10.1002/adma.201604634.

97. Finnie KS, Waller DJ, Perret FL, Krause-Heuer AM, Lin HQ, Hanna JV, et al. Biodegradability of sol-gel silica microparticles for drug delivery. J Sol-Gel Sci Technol. 2009;49(1):12-8 http:// link.springer.com/10.1007/s10971-008-1847-4.

98. Chen G, Teng Z, Su X, Liu Y, Lu G. Unique biological degradation behavior of stöber mesoporous silica nanoparticles from their interiors to their exteriors. J Biomed Nanotechnol. 2015;11(4):722-9 http://openurl.ingenta.com/content/xref? genre $=$ article $\&$ issn $=1550-7033 \&$ volume $=11 \&$ issue $=4 \&$ spage $=$ 722.

99. Huang X, Li L, Liu T, Hao N, Liu H, Chen D, et al. The shape effect of mesoporous silica nanoparticles on biodistribution, clearance, and biocompatibility in vivo. ACS Nano. 201 1;5(7):5390-9. https://doi.org/10.1021/nn200365a.

100. He Q, Shi J, Zhu M, Chen Y, Chen F. The three-stage in vitro degradation behavior of mesoporous silica in simulated body fluid. Microporous Mesoporous Mater. 2010;131(1-3):314-20 https:// linkinghub.elsevier.com/retrieve/pii/S1387181110000107.

101. Li L, Liu T, Fu C, Tan L, Meng X, Liu H. Biodistribution, excretion, and toxicity of mesoporous silica nanoparticles after oral administration depend on their shape. Nanomedicine Nanotechnology, Biol Med. 2015;11(8):1915-24 https:// linkinghub.elsevier.com/retrieve/pii/S1549963415001471.

102. Hao N, Liu H, Li L, Chen D, Li L, Tang F. In vitro degradation behavior of silica nanoparticles under physiological conditions. J Nanosci Nanotechnol. 2012;12(8):6346-54 http://www. ingentaconnect.com/content/10.1166/jnn.2012.6199.

103. Ehrlich H, Demadis KD, Pokrovsky OS, Koutsoukos PG. Modern views on desilicification: biosilica and abiotic silica dissolution in natural and artificial environments. Chem Rev. 2010;110(8):4656-89. https://doi.org/10.1021/cr900334y.

104. He Q, Zhang Z, Gao F, Li Y, Shi J. In vivo biodistribution and urinary excretion of mesoporous silica nanoparticles: effects of 
particle size and PEGylation. Small. 2011;7(2):271-80. https:// doi.org/10.1002/smll.201001459.

105. Burns AA, Vider J, Ow H, Herz E, Penate-Medina O, Baumgart $\mathrm{M}$, et al. Fluorescent silica nanoparticles with efficient urinary excretion for nanomedicine. Nano Lett. 2009;9(1):442-8. https://doi.org/10.1021/nl803405h.

106. Kumar R, Roy I, Ohulchanskky TY, Vathy LA, Bergey EJ, Sajjad $\mathrm{M}$, et al. In vivo biodistribution and clearance studies using multimodal organically modified silica nanoparticles. ACS Nano. 2010;4(2):699-708. https://doi.org/10.1021/nn901146y.

107. Cho M, Cho W-S, Choi M, Kim SJ, Han BS, Kim SH, et al. The impact of size on tissue distribution and elimination by single intravenous injection of silica nanoparticles. Toxicol Lett. 2009;189(3):177-83 https://linkinghub.elsevier.com/retrieve/ pii/S0378427409002136.

108. Sarparanta M, Bimbo LM, Rytkönen J, Mäkilä E, Laaksonen TJ, Laaksonen $\mathrm{P}$, et al. Intravenous delivery of hydrophobinfunctionalized porous silicon nanoparticles: stability, plasma protein adsorption and biodistribution. Mol Pharm. 2012;9(3):654 63. https://doi.org/10.1021/mp20061ld.

109. Souris JS, Lee C-H, Cheng S-H, Chen C-T, Yang C-S, Ho JA, et al. Surface charge-mediated rapid hepatobiliary excretion of mesoporous silica nanoparticles. Biomaterials. 2010;31(21): 5564-74 https://linkinghub.elsevier.com/retrieve/pii/ S0142961210004199.

110. Subramaniam, Thomas, Gustafsson, Jambhrunkar, Kidd, Prestidge. Rifampicin-loaded mesoporous silica nanoparticles for the treatment of intracellular infections. Antibiotics. 2019;8(2):39. https://www.mdpi.com/2079-6382/8/2/39

111. Martínez-Carmona M, Izquierdo-Barba I, Colilla M, Vallet-Regí M. Concanavalin A-targeted mesoporous silica nanoparticles for infection treatment. Acta Biomater. 2019;96:547-56 https:// linkinghub.elsevier.com/retrieve/pii/S1742706119304817.

112. Deaconu M, Pintilie L, Vasile E, Mitran R-A, Gradisteanu Pircalabioru G, Matei C, et al. Norfloxacin delivery systems based on MCM-type silica carriers designed for the treatment of severe infections. Mater Chem Phys. 2019;238:121886 https:// linkinghub.elsevier.com/retrieve/pii/S0254058419306832.

113. Yang S, Han X, Yang Y, Qiao H, Yu Z, Liu Y, et al. Bacteriatargeting nanoparticles with microenvironment-responsive antibiotic release to eliminate intracellular Staphylococcus aureus and associated infection. ACS Appl Mater Interfaces. 2018;10(17): 14299-311. https://doi.org/10.1021/acsami.7b15678.

114. Zhou X, Weng W, Chen B, Feng W, Wang W, Nie W, et al. Mesoporous silica nanoparticles/gelatin porous composite scaffolds with localized and sustained release of vancomycin for treatment of infected bone defects. J Mater Chem B. 2018;6(5):740-52 http://xlink.rsc.org/?DOI=C7TB01246B.

115. Xu C, He Y, Li Z, Ahmad Nor Y, Ye Q. Nanoengineered hollow mesoporous silica nanoparticles for the delivery of antimicrobial proteins into biofilms. J Mater Chem B. 2018;6(13):1899-902 http://xlink.rsc.org/?DOI=C7TB03201C.

116. Deaconu M, Nicu I, Tincu R, Brezoiu A-M, Mitran R-A, Vasile $\mathrm{E}$, et al. Tailored doxycycline delivery from MCM-41-type silica carriers. Chem Pap. 2018;72(8):1869-80 http://link.springer. com/10.1007/s1 1696-018-0457-z.

117. Andrade GF, Faria JAQA, Gomes DA, de Barros ALB, Fernandes RS, Coelho ACS, et al. Mesoporous silica SBA-16/ hydroxyapatite-based composite for ciprofloxacin delivery to bacterial bone infection. J Sol-Gel Sci Technol. 2018;85(2):369-81 http://link.springer.com/10.1007/s10971-017-4557-y.

118. Chen W, Cheng C-A, Lee B-Y, Clemens DL, Huang W-Y, Horwitz MA, et al. Facile strategy enabling both high loading and high release amounts of the water-insoluble drug clofazimine using mesoporous silica nanoparticles. ACS Appl Mater
Interfaces. 2018;10(38):31870-81. https://doi.org/10.1021/ acsami.8b09069.

119. González B, Colilla M, DíezJ, Pedraza D, Guembe M, IzquierdoBarba I, et al. Mesoporous silica nanoparticles decorated with polycationic dendrimers for infection treatment. Acta Biomater. 2018;68:261-71 https://linkinghub.elsevier.com/retrieve/pii/ S1742706117308073.

120. Cao C, Ge W, Yin J, Yang D, Wang W, Song X, et al. Mesoporous silica supported silver-bismuth nanoparticles as photothermal agents for skin infection synergistic antibacterial therapy. Small. 2020;2000436. https://doi.org/10.1002/smll. 202000436.

121. Tasia W, Lei C, Cao Y, Ye Q, He Y, Xu C. Enhanced eradication of bacterial biofilms with DNase I-loaded silver-doped mesoporous silica nanoparticles. Nanoscale. 2020;12(4):2328-32 http:// xlink.rsc.org/?DOI=C9NR08467C.

122. $\mathrm{Xu} \mathrm{T,} \mathrm{LiJ,} \mathrm{Zhang} \mathrm{S,} \mathrm{Jin} \mathrm{Y,} \mathrm{Wang} \mathrm{R.} \mathrm{Integration} \mathrm{of} \mathrm{diagnosis} \mathrm{and}$ treatment in the detection and kill of S.aureus in the whole blood. Biosens Bioelectron. 2019;142:111507 https://linkinghub. elsevier.com/retrieve/pii/S095656631930586X.

123. Ding Y, Hao Y, Yuan Z, Tao B, Chen M, Lin C, et al. A dualfunctional implant with an enzyme-responsive effect for bacterial infection therapy and tissue regeneration. Biomater Sci. 2020;8(7): 1840-54 http://xlink.rsc.org/?DOI=C9BM01924C.

124. Encinas N, Angulo M, Astorga C, Colilla M, Izquierdo-Barba I, Vallet-Regí M. Mixed-charge pseudo-zwitterionic mesoporous silica nanoparticles with low-fouling and reduced cell uptake properties. Acta Biomater. 2019;84:317-27 https://linkinghub. elsevier.com/retrieve/pii/S1742706118307372.

125. Clemens DL, Lee B-Y, Plamthottam S, Tullius MV, Wang R, Yu C-J, et al. Nanoparticle formulation of moxifloxacin and intramuscular route of delivery improve antibiotic pharmacokinetics and treatment of pneumonic tularemia in a mouse model. ACS Infect Dis. 2019;5(2):281-91. https://doi.org/10.1021/acsinfecdis. $8 \mathrm{~b} 00268$.

126. Gounani Z, Asadollahi MA, Meyer RL, Arpanaei A. Loading of polymyxin B onto anionic mesoporous silica nanoparticles retains antibacterial activity and enhances biocompatibility. Int J Pharm. 2018;537(1-2):148-61 https://linkinghub.elsevier.com/retrieve/ pii/S0378517317311833.

127. Abdelbar MF, Shams RS, Morsy OM, Hady MA, Shoueir K, Abdelmonem R. Highly ordered functionalized mesoporous silicate nanoparticles reinforced poly (lactic acid) gatekeeper surface for infection treatment. Int J Biol Macromol. 2020;156:858-68 https: / / linkinghub.elsevier.com/retrieve/pii/ S0141813020329846.

128. Michailidis M, Sorzabal-Bellido I, Adamidou EA, DiazFernandez YA, Aveyard J, Wengier R, et al. Modified mesoporous silica nanoparticles with a dual synergetic antibacterial effect. ACS Appl Mater Interfaces. 2017;9(44):38364-72. https://doi. org/10.1021/acsami.7b14642.

129. Vale N, Correia A, Silva S, Figueiredo P, Mäkilä E, Salonen J, et al. Preparation and biological evaluation of ethionamidemesoporous silicon nanoparticles against Mycobacterium tuberculosis. Bioorg Med Chem Lett. 2017;27(3):403-5 https:// linkinghub.elsevier.com/retrieve/pii/S0960894X16313397.

130. Lee B-Y, Li Z, Clemens DL, Dillon BJ, Hwang AA, Zink JI, et al. Redox-triggered release of moxifloxacin from mesoporous silica nanoparticles functionalized with disulfide snap-tops enhances efficacy against pneumonic tularemia in mice. Small. 2016;12(27): 3690-702. https://doi.org/10.1002/smll.201600892.

131. Hao N, Chen X, Jeon S, Yan M. Carbohydrate-conjugated hollow oblate mesoporous silica nanoparticles as nanoantibiotics to target mycobacteria. Adv Healthc Mater. 2015;4(18):2797801. https://doi.org/10.1002/adhm.201500491. 
132. Hwang AA, Lee B-Y, Glemens DL, Dillon BJ, Zink JI, Horwitz MA. pH-Responsive isoniazid-loaded nanoparticles markedly improve Tuberculosis treatment in mice. Small. 2015;11(38):506678. https://doi.org/10.1002/smll.201500937.

133. Clemens DL, Lee B-Y, Xue M, Thomas CR, Meng H, Ferris D, et al. Targeted intracellular delivery of antituberculosis drugs to mycobacterium tuberculosis-infected macrophages via functionalized mesoporous silica nanoparticles. Antimicrob Agents Chemother. 2012;56(5):2535-45 https://aac.asm.org/content/ $56 / 5 / 2535$

134. Li Z, Clemens DL, Lee B-Y, Dillon BJ, Horwitz MA, Zink JI. Mesoporous silica nanoparticles with ph-sensitive nanovalves for delivery of moxifloxacin provide improved treatment of lethal pneumonic tularemia. ACS Nano. 2015;9(11):10778-89. https://doi.org/10.1021/acsnano.5b04306.

135. Ruehle B, Clemens DL, Lee B-Y, Horwitz MA, Zink JI. A Pathogen-specific cargo delivery platform based on mesoporous silica nanoparticles. J Am Chem Soc. 2017;139(19):6663-8. https://doi.org/10.1021/jacs. 7b01278.

136. Oliveira DC de P, de Barros ALB, Belardi RM, de Goes AM, de Oliveira Souza BK, Soares DCF. Mesoporous silica nanoparticles as a potential vaccine adjuvant against Schistosoma mansoni. J Drug Deliv Sci Technol 2016;35:234-240. https://linkinghub. elsevier.com/retrieve/pii/S177322471630106X

137. Nhavene EPF, da Silva WM, Trivelato Junior RR, Gastelois PL, Venâncio T, Nascimento R, et al. Chitosan grafted into mesoporous silica nanoparticles as benznidazol carrier for Chagas diseases treatment. Microporous Mesoporous Mater. 2018;272:265-75 https:/ / linkinghub.elsevier.com/retrieve/pii/ S1387181118303457.

138. Tawfeek GM, Baki MHA, Ibrahim AN, Mostafa MAH, Fathy MM, Diab MSEDM. Enhancement of the therapeutic efficacy of praziquantel in murine Schistosomiasis mansoni using silica nanocarrier. Parasitol Res. 2019;118(12):3519-33 http://link. springer.com/10.1007/s00436-019-06475-8.

139. Yunessnia lehi A, Shagholani H, Nikpay A, Ghorbani M, Soleimani lashkenari M, Soltani M. Synthesis and modification of crystalline SBA-15 nanowhiskers as a $\mathrm{pH}$-sensitive metronidazole nanocarrier system. Int J Pharm. 2019;555:28-35. https:// linkinghub.elsevier.com/retrieve/pii/S0378517318308524

140. Montazeri M, Razzaghi-Abyaneh M, Nasrollahi SA, Maibach H, Nafisi S. Enhanced topical econazole antifungal efficacy by aminefunctionalized silica nanoparticles. Bull Mater Sci. 2020;43(1):13 http://link.springer.com/10.1007/s12034-019-1974-2.

141. Paramanantham P, Antony AP, Sruthil Lal SB, Sharan A, Syed A, Ahmed M, et al. Antimicrobial photodynamic inactivation of fungal biofilm using amino functionalized mesoporus silica-rose bengal nanoconjugate against Candida albicans. Sci African. 2018;1: e00007 https://linkinghub.elsevier.com/retrieve/pii/ S246822761830098X.

142. Mas N, Galiana I, Hurtado S, Mondragón L, Bernardos A, Sancenón F, et al. Enhanced antifungal efficacy of tebuconazole using gated $\mathrm{pH}$-driven mesoporous nanoparticles. Int $\mathrm{J}$ Nanomedicine. 2014;9(1):2597-606 http://www.dovepress. com/enhanced-antifungal-efficacy-of-tebuconazole-using-gatedph-driven-mes-peer-reviewed-article-IJN.

143. Mitra S, Patra P, Pradhan S, Debnath N, Dey KK, Sarkar S, et al. Microwave synthesis of $\mathrm{ZnO} @ \mathrm{mSiO}_{2}$ for detailed antifungal mode of action study: Understanding the insights into oxidative stress. J Colloid Interface Sci. 2015;444:97-108 https:// linkinghub.elsevier.com/retrieve/pii/S0021979714009941.

144. Qasim M, Singh BR, Naqvi AH, Paik P, Das D. Silver nanoparticles embedded mesoporous $\mathrm{SiO}_{2}$ nanosphere: an effective anticandidal agent against Candida albicans 077. Nanotechnology. 2015;26(28):285102 https://iopscience.iop.org/article/10.1088/ 0957-4484/26/28/285102.
145. LaBauve AE, Rinker TE, Noureddine A, Serda RE, Howe JY, Sherman MB, et al. Lipid-coated mesoporous silica nanoparticles for the delivery of the ML336 antiviral to inhibit encephalitic alphavirus infection. Sci Rep. 2018;8(1):13990 http://www. nature.com/articles/s41598-018-32033-w.

146. Lee EC, Davis-Poynter N, Nguyen CTH, Peters AA, Monteith GR, Strounina E, et al. GAG mimetic functionalised solid and mesoporous silica nanoparticles as viral entry inhibitors of herpes simplex type 1 and type 2 viruses. Nanoscale. 2016;8(36):16192-6 http://xlink.rsc.org/?DOI=C6NR03878F.

147. Lee EC, Nguyen CTH, Strounina E, Davis-Poynter N, Ross BP. Structure-activity relationships of GAG mimetic-functionalized mesoporous silica nanoparticles and evaluation of acyclovirloaded antiviral nanoparticles with dual mechanisms of action. ACS Omega. 2018;3(2):1689-99. https://doi.org/10.1021/ acsomega.7b01662.

148. Guo H-C, Feng X-M, Sun S-Q, Wei Y-Q, Sun D-H, Liu X-T, et al. Immunization of mice by hollow mesoporous silica nanoparticles as carriers of porcine circovirus type 2 ORF2 protein. Virol J. 2012;9(1):108 http://virologyj.biomedcentral.com/ articles/10.1186/1743-422X-9-108.

149. Rodrigues GR, López-Abarrategui C, de la Serna GI, Dias SC, Otero-González AJ, Franco OL. Antimicrobial magnetic nanoparticles based-therapies for controlling infectious diseases. Int $\mathrm{J}$ Pharm. 2019;555:356-67 https://linkinghub.elsevier.com/ retrieve/pii/S0378517318308688.

150. Walvekar P, Gannimani R, Govender T. Combination drug therapy via nanocarriers against infectious diseases. Eur J Pharm Sci. 2019;127:121-41 https://linkinghub.elsevier.com/retrieve/pii/ S09280987 18304640.

151. Singh A, Gautam PK, Verma A, Singh V, Shivapriya PM, Shivalkar S, et al. Green synthesis of metallic nanoparticles as effective alternatives to treat antibiotics resistant bacterial infections: A review. Biotechnol Reports. 2020;25:e0427.

152. Abdullah MAIA, Al Dajah S, Murad Aabu, El-Salem AM, Khafajah ALIM. Current Research in Nutrition and Food Science. 2019.

153. Lee JH, Jeong SH, Cha S-S, Lee SH. A lack of drugs for antibiotic-resistant Gram-negative bacteria. Nat Rev Drug Discov. 2007;6(11):938-8 http://www.nature.com/articles/ nrd2201-c1.

154. Yenkoidiok-Douti L, Jewell GM. Integrating biomaterials and immunology to improve vaccines against infectious diseases. ACS Biomater Sci Eng. 2020;6(2):759-78. https://doi.org/10.1021/ acsbiomaterials.9b01255.

155. Douglas LJ. Candida biofilms and their role in infection. Trends Microbiol. 2003;11(1):30-6 https://linkinghub.elsevier.com/ retrieve/pii/S0966842X02000021

156. Firooz A, Nafisi S, Maibach HI. Novel drug delivery strategies for improving econazole antifungal action. Int J Pharm. 2015;495(1): 599-607 https://linkinghub.elsevier.com/retrieve/pii/ S0378517315302131.

157. Descalzo AB, Martínez-Máñez R, Sancenón F, Hoffmann K, Rurack K. The supramolecular chemistry of organic-inorganic hybrid materials. Angew Chemie Int Ed. 2006;45(36):5924-48. https://doi.org/10.1002/anie.200600734.

158. Nasrollahzadeh M, Sajjadi M, Soufi GJ, Iravani S, Varma RS. Nanomaterials and nanotechnology-associated innovations against viral infections with a focus on Coronaviruses. Nanomaterials. 2020;10(6):1072 https://www.mdpi.com/20794991/10/6/1072.

159. Nikaeen G, Abbaszadeh S, Yousefinejad S. Application of nanomaterials in treatment, anti-infection and detection of coronaviruses. Nanomedicine. 2020;15(15):1501-12. /pmc/articles/ PMC7373208/?report=abstract 
160. Farjadian F, Roointan A, Mohammadi-Samani S, Hosseini M. Mesoporous silica nanoparticles: Synthesis, pharmaceutical applications, biodistribution, and biosafety assessment. Chem Eng J. 2019;359:684-705 https://linkinghub.elsevier.com/retrieve/pii/ S138589471832391X.

161. Kankala RK, Han Y, Na J, Lee C, Sun Z, Wang S, et al. Nanoarchitectured structure and surface biofunctionality of mesoporous silica nanoparticles. Adv Mater. 2020;32(23):1907035. https://doi.org/10.1002/adma.201907035.

162. Benezra M, Penate-Medina O, Zanzonico PB, Schaer D, Ow H, Burns A, et al. Multimodal silica nanoparticles are effective cancer-targeted probes in a model of human melanoma. J Clin Invest. 2011 ; 121(7):2768-80 http://www.jci.org.

163. Phillips E, Penate-Medina O, Zanzonico PB, Carvajal RD, Mohan $\mathrm{P}$, Ye Y, et al . Clinical translation of an ultrasmall inorganic optical-PET imaging nanoparticle probe. Sci Transl Med. 2014;6(260):260ra149. https://stm.sciencemag.org/content/6/ 260/260ral49

164. Bradbury MS, Pauliah M, Zanzonico P, Wiesner U, Patel S. Intraoperative mapping of sentinel lymph node metastases using a clinically translated ultrasmall silica nanoparticle. Wiley Interdiscip Rev Nanomedicine Nanobiotechnology. 2016;8(4): 535-53. https://doi.org/10.1002/wnan.1380.

165. Bradbury MS, Phillips E, Montero PH, Cheal SM, Stambuk H, Durack JC, et al. Clinically-translated silica nanoparticles as dualmodality cancer-targeted probes for image-guided surgery and interventions. Integr Biol. 2013;5(1):74-86 https://academic. oup.com/ib/article/5/1/74/5208312.

166. Chen F, Ma K, Zhang L, Madajewski B, Zanzonico P, Sequeira S, et al. Target-or-clear zirconium-89 labeled silica nanoparticles for enhanced cancer-directed uptake in melanoma: A comparison of radiolabeling strategies. Chem Mater. 2017;29(19):8269-81 https://pubs.acs.org/sharingguidelines.

167. Kharlamov AN, Gabinsky JL. Plasmonic photothermic and stem cell therapy of atherosclerotic plaque as a novel nanotool for angioplasty and artery remodeling. Rejuvenation Res. 2012;15(2): 222-30 https://pubmed.ncbi.nlm.nih.gov/22533437/.
168. Kharlamov AN, Tyurnina AE, Veselova VS, Kovtun OP, Shur VY, Gabinsky JL. Silica-gold nanoparticles for atheroprotective management of plaques: Results of the NANOM-FIM trial. Nanoscale. 2015;7(17):8003-15 https://pubs.rsc.org/en/ content/articlehtml/2015/nr/c5nr01050k.

169. Bobo D, Robinson KJ, Islam J, Thurecht KJ, Corrie SR. Nanoparticle-based medicines: A review of FDA-approved materials and clinical trials to date. Pharmaceutical Research. 2016;33: 2373-87 https://link.springer.com/article/10.1007/s1 1095016-1958-5.

170. Dogra P, Adolphi NL, Wang Z, Lin Y-S, Butler KS, Durfee PN, et al. Establishing the effects of mesoporous silica nanoparticle properties on in vivo disposition using imaging-based pharmacokinetics. Nat Commun. 2018;9(1):4551 www.nature.com/ naturecommunications.

171. Lei Q Guo J, Noureddine A, Wang A, Wuttke S, Brinker GJ, et al. Sol-gel-based advanced porous silica materials for biomedical applications. Adv Funct Mater. 2020;1909539. https://doi. org/10.1002/adfm.201909539.

172. Kim C, Yoon S, Lee JH. Facile large-scale synthesis of mesoporous silica nanoparticles at room temperature in a monophasic system with fine size control. Microporous Mesoporous Mater. 2019;288:109595 https://linkinghub.elsevier.com/retrieve/pii/ S1387181119304524.

173. Š Soltys M, Balouch M, Kašpar O, Lhotka M, Ulbrich P, Zadražil A, et al. Evaluation of scale-up strategies for the batch synthesis of dense and hollow mesoporous silica microspheres. Chem Eng J. 2018;334:1135-47 https://linkinghub.elsevier.com/retrieve/pii/ S1385894717319344.

174. Kankala RK, Zhang H, Liu C, Kanubaddi KR, Lee C, Wang S, et al. Metal species-encapsulated mesoporous silica nanoparticles: current advancements and latest breakthroughs. Adv Funct Mater. 2019;29(43):1902652. https://doi.org/10.1002/adfm. 201902652.

Publisher's Note Springer Nature remains neutral with regard to jurisdictional claims in published maps and institutional affiliations. 